\title{
SIGNIFICANCE AND DISTRIBUTION OF THE MIC A/B ALLELES AMONG THE WORLDWIDE POPULATIONS
}

\author{
Baburajan Radha $^{{ }^{1}}$, Manimaran Nellai Chinniah ${ }^{2}$ and Gnanaraj Muniraj ${ }^{3}$ \\ ${ }^{1}$ MVK Hospital- Hitech Diagnostic laboratory, Tanjavur-02, Tamil Nadu, India. \\ ${ }^{2}$ Network Resource Centre for Biological Sciences, School of Biological Sciences, Madurai Kamaraj University, \\ Madurai-21,Tamil Nadu, India. \\ ${ }^{3}$ Department of Plant Morphology and Algology, School of Biological Sciences, Madurai Kamaraj University, \\ Madurai-21,Tamil Nadu, India.
}

\section{ABSTRACT}

The Major histocompatibility complex chain 1(MIC) genes are recently studied upon their association with various infections, Autommune and Inflammatory diseases, various cancers and Graft rejections. The expression of these genes mediates numerous key cellular and immunological pathways. Numerous alleles are reported for these genes across the worldwide populations. This report utilizes the public domain data in effectively analyzing the various MIC allelic distributions in different population subgroups in the light of previous reports and established hypotheses in the context of clinical and population genetics.

\section{KEY WORDS}

HLAs, Allele frequencies, Racial Admixture and Genetic predisposition.

\section{INTRODUCTION:}

Antigen presenting systems such as the Major histocompatibility complex (MHC) are relatively primitive innate immune mechanisms and are conserved across numerous species [1-10]. The human leukocyte antigens (HLAs) are human MHC and are present in the short arm region of Chromosome 6.They are reported to be mediating a plethora of immune regulations. The genetic variations of this region were studied for decades, in respect to tissue transplantation and association with various clinical complications and autoimmune and infectious diseases [11-16]. HLAs are also highly polymorphic and reported to be ethnicity-specific, hence studied in the context of human evolution and ethno-specific susceptibility or resistance towards diseases [17-23].

Another interesting group of genes mapped in the Chromosome 6, called the Major histocompatibility complex class 1 chain-related (MIC) genes (Figure A), are recently reported [24], in the similar context as that of HLAs. The MIC comprises MHC class I polypeptide-related sequence $A$ (MICA) and $\mathrm{MHC}$ class I polypeptide-related sequence $B$ (MICB).The sequence of MICA is highly polymorphic with more than 100 identified alleles as reported in http://www.ebi.ac.uk/ipd/imgt/hla/.

The products of these genes, the MIC A/B proteins are expressed in the gastrointestinal epithelium [25].They are ligands to the NKG2D receptor of natural killer (NK) cells and are primarily regulated by the NF-kB $[26,27]$. This receptor-ligand interaction result in the production of cytokines and subsequent cytolysis of target cells. The mechanism of shedding of these ligands by the tumour cells, in order to escape cytolysis is an intriguing phenomenon. Hence, various approaches were devised to upregulate the MIC expression in the target cells, which would in turn increase the cytotoxicity in tumors [28].

Recently, the polymorphisms and expression of the MHC class I chain(MICA/B)-related alleles are reported to be associated with various complications such as Psoriasis [29], Ocular toxoplasmosis[30], Behçet's disease [31], Alcoholic 
Liver disease [32], Liver fibrosis [33], Ankylosing spondylitis [34], Celiac disease [35], HIV infections [36], Autoimmune diseases[37-39], and importantly, with Graft rejection [40, 41], and numerous cancers[42-47].
Such MIC variations are widely reported in worldwide populations such as Chinese [33, 48, 49, 50], Indian [51], Iranian [52, 53], Swedish [54], Finnish [55], Korean [56, 57], Spanish [58], Bolivian [59], and Algerian [60].

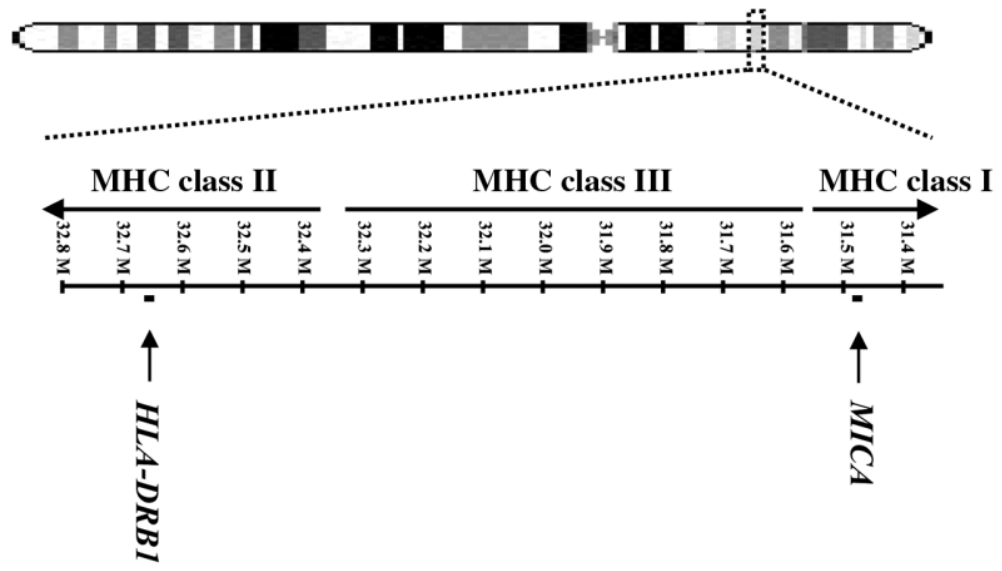

Figure A: Genetic arrangement of the MIC genes within the MHC genes.

\section{DATA SELECTION AND ANALYSES:}

The allelic frequency data of various MIC genes for around 30 worldwide populations were collected from the public open-access database www.Allelefrequencies.net (Tables 1-7).The frequencies of individual alleles to the corresponding populations were calculated using MS-Excel. Neighbor Joining (NJ) Tree (Figure: 32A) and Principal component analysis (PCA) (Figure: 32B) were performed to identify possible clustering among the populations using the PAST (Paleontological statistics) software [61].Self organizing maps (SOM) were constructed using the unsupervised heuristic approaches to find the presence of possible hidden patterns. For this purpose, we used the $\mathrm{R}$ package 'kohonen'[62]. Only informative alleles were considered.16 nodes (neurons) were used. Number of Iterations=1000.n.hood=Circular. alpha=Guessing statistical parameter (FIGURES:33-61).

\section{RESULTS AND DISCUSSION:}

All the allelic frequencies of both $M I C A / B$ gene (Columns) against the corresponding populations (Rows) are depicted in the matrix plot (Figure
1).According to their class, the alleles were tabulated with their respective populations (Figure 2-29).

In this study, various Asian, South American and African and Afro-American populations are observed to be completely devoid of all the $A$ group (A4-A10) alleles (Figures: 1 and 2-5). The Asian study populations, except the Japanese, comprised the MICB alleles (MICB*001 to $\left.M I C B^{*} 029\right)$, which are completely absent in all other world populations (Figures: 1 and 6-13).In addition, the alleles MICA *007:02, MICA *013 and MICA *014 are observed to be totally absent in all the study populations (Figures: 1 and 14-21).

The A5 allele is widely distributed across the various Asian, Middle-East and Southern European populations (Figures: 1 and 2-5). This allele is reported to be associated with Liver fibrosis in Chinese communities [33]. Meanwhile, the Malaysian Chinese community is reported to be susceptible to Nasopharyngeal Carcinoma due to the MICA variations [64].

The MHC SNPs rs76546355, rs2848713, rs76546355 and rs2848713 which are in close proximity with MICA are associated with Behcet's 
disease in the Iranian community [52]. In addition, the $M I C B$ expression is reported to be associated with susceptibility towards Multiple sclerosis in this population [53]. Interestingly, this current study does not indicate any presence of $M I C A / B$ allelic variations in Iranian population.

The MICA *011 allele is reported [59] to be associated with protection against Chronic Chagas Disease among the Bolivian subjects. Even though, this study does not include Bolivian subjects, the neighbor communities could be considered for possible presence of this allele. Evidently, the USA Caucasian population 2, Brazilian, Argentinean and the Spanish Majorca Chueta populations (Figures: 1 and 18-21) possess this allele, albeit in low frequencies.

MICA *009 alleles are associated, though in statistically insignificant levels, with Cutaneous malignant melanoma in South eastern Spanish communities [58] .This present study involves the MICA *009 alleles with two other subtypes, MICA *009:01 and MICA*009:02. The MICA *009 allele is observed in Majorca Chueta Spanish, North East Thais, USA Caucasian population 2 and Japanese populations (Figures: 1 and 14-17). Meanwhile, the MICA *009:01 allele is found to be in Baotou Han Chinese and various South American populations. It is found in the Brazilian subgroups such as Mato Grosso do Sul Nandeva, Parana Kaingang, Parana Guarani M'byá, Mato Grosso do Sul Kaiowa and Limao Verde- Corrego Reserve Terena, and in the Argentinean subgroups such as Formosa Wichi and Formosa Toba. The Moroccan subgroup Nador Metalsa also has this allele in high frequency (Figures: 1 and 14-17). The MICA*009:02 is observed in the Formosa Toba Argentinean, Moroccan Nador Metalsa Moroccan, Nigerian subgroups such as South Efik and South Yoruba and in the USA Boston Afro-American subgroups (Figures: 1 and 14-17). The Baotou Han Chinese is the only Asian community with both MICA*009:01 and MICA*009:02.

The allele MICA *012:01 is observed only in the Brazilian Parana Kaingang population subgroup

(Figures: 1 and 19). Meanwhile, the SOMs of both MICA *12:01(Figure: 46) and MICA *16 (Figure: 48) exhibit similar pattern. Another pattern is shared among the alleles MICA *17(FIGURE: 49), MICA*19 (Figure: 51), $M I C A * 52$ (Figure: 56) and $M I C B$ *13(Figure: 60). In addition, the alleles MICB *02.1.1 (Figure: 57) and MICB *009N (Figure: 59) also exhibit similar SOM pattern.

The MICA *027 allele is found to be absent in all populations, but observed only in the Spanish Murcia and various Brazilian and Argentinean communities (Figures: 1 and 22-25).One possibility for this interesting distribution (Figure: 30 ) could be the admixture of Spaniards with the South American natives during the discovery of the Americas. The allele MICA *046 is observed only among the Morocco Nador Metalsa and Spain Murcia populations (Figures: 1 and 26-29). The Morocco Nador Metalsa is also the only population with the MICA *035 allele (Figures: 1 and 27).

Strikingly, the MICA *041 allele is observed only among the native African and Afro-American populations such as Nigerian South Yoruba, USA African-American pop 2, Nigerian South Igbo, Nigerian South Efik and USA Boston AfricanAmerican ( Figures:1,26-29 and 31) .This allele is observed among no other world populations. This allele seems much restricted to the African ethnicity and not shared among the other populations, despite the 400 years of racial admixture, since the discovery of the New World.

In the PCA analysis, almost all the Asian populations were observed to be clustered together. Also clustered were the groups of Middle-Eastern, Southern European, African and Afro-American and South American populations. Interestingly, the USA Caucasian population (Pop 2 ) is found to be clustered along with the Asian populations. The ungrouped populations were found to be the Chinese Bautou Han, Spanish Murcia and Spanish Majorca Chueta populations (Figure 32B).Almost similar groupings were observed in the NJ tree analysis (Figure 32A).

The allele MICA *002 is reportedly involved with Ocular Taxoplasmosis [30] . In this study, the MICA *02 is observed among the Spanish subgroups such as Murcia, Majorca (high frequencies) and Majorca Chueta, the USA Caucasian pop2 and 
Asian communities such as the North East Taiwanese subgroup and the Japanese (Figures:1 and 14-17). The allelic subtype MICA *002:01 is observed to be widely distributed among the Brazilian subgroups such as Parana Kaingang, Parana Guarani M'byá, Mato Grosso do Sul Kaiowa , Limao Verde and Corrego Reserve Terena, Argentinean communities such as Formosa Wichi, and Formosa Toba, Nigerian subgroups such as South Efik and South Igbo and South Yoruba, Moroccan Nador Metalsa, the USA Boston African American and the USA African American pop 2 (Figures:1 and 14-17). Another subtype MICA *02:02 is observed among the Baotou Han Chinese and the Mato Grosso do Sul Nandeva Brazilian (high frequencies) subgroups (Figures:1 and 1417).

The allele MICA *007 is associated with the elicitation of inflammatory cascade via fibroblasts [37]. An allelic subtype MICA*007:01 is also implicated in the pathogenesis of Ankylosing Spondylitis in both Caucasian and Chinese subjects [34]. In this study, the MICA *007 allele is observed among the Spanish subgroups such as Murcia, Majorca and Majorca Chueta, the USA Caucasian population 2 and the Japanese. The subtype MICA*007:01 is observed among the Baotou Han chinese, South American communities such as Mato Grosso do Sul Nandeva Brazilian, and Formosa Wichi Argentinean and the Nador Metalsa Moroccan subgroups (Figures:1 and 1417).

The allele MICA *008 is reported to be associated with Inflammatory bowel disease (IBD) and Peripheral Arthropathy [64], Allosensitization in Kidney transplants[41] and fibroblast inflammatory pathways[37].This current study details the presence of this allele among the Spanish communities such as Murcia, Majorca and Majorca Chueta, North East Thais, USA Caucasian population 2 and the Japanese communities. The subtype, MICA *008:01 is observed among the Baotou Han Chinese, Brazilian subgroups such as Mato Grosso do Sul Nandeva, Parana Kaingang, Parana Guarani M'byá, Mato Grosso do Sul Kaiowa and Limao Verde and Corrego Reserve Terena, Nador Metalsa Moroccan, South Efik Nigerian, Formosa Toba Argentinean and the USA Boston African American communities. Another subtype, MICA *008:02- Baotou Han Chinese, USA Boston African American, USA African American population 2 and Nigerian communities such as South Efik, South Yoruba and South Igbo (Figures:1 and 14-17).

The allele MICA * 17 confers protection against HIV infections[36]. This allele is observed among the Spanish subgroups such as Murcia and Majorca Chueta, Nador Metalsa Morrocan, and North East Thais in this study (Figures: 1 and 18-21).

The allele MICA*019 is associated with the risk of Ankylosing spondylitis in Chinese populations[34].This allele is also reportedly associated with the onset of inflammation via fibroblasts[37]. This is allele is observed among the Mato Grosso do Sul Nandeva Brazilian, Majorca Chueta Spanish, Nador Metalsa Moroccan, North East Thais and the Japanese communities. Interestingly, no Chinese populations are observed to be possessing this allele in this present study (Figures: 1 and 18-21).

\section{4 .LIMITATIONS:}

One of the obvious limitations is that the MIC alleles are yet to be reported in larger cohorts in evolutionarily informative populations such as Indian, Scandinavian, Native American, Spanish Basque, Andamanese and Taiwanese, etc. Studies with other minor populations such as Korean, Western European, Turkish and Slavic communities also could facilitate effective inferences. These MIC alleles are reportedly more effective when present as haplotypes with HLAs and other key genes, rather than acting alone. This is the primary limitation of the present study, as it considers only the distribution of MIC alleles alone among the world populations. This analysis is also statistically limited by the different sample numbers of the populations, the allele frequencies were calculated from. 


\begin{tabular}{|c|c|c|c|c|c|c|}
\hline & A4 & A5 & A5.1 & A6 & A9 & A10 \\
\hline China Baotou Han & 0.115 & 0.293 & 0.236 & 0.178 & 0.139 & \\
\hline China Guangzhou Han & 0.132 & 0.288 & 0.264 & 0.146 & 0.17 & \\
\hline China Hui & 0.091 & 0.307 & 0.239 & 0.17 & 0.193 & \\
\hline China Hunan Province Han & 0.147 & 0.376 & 0.265 & 0.076 & 0.118 & 0.006 \\
\hline China North Han & 0.094 & 0.281 & 0.266 & 0.156 & 0.203 & \\
\hline China Uygur & 0.071 & 0.163 & 0.225 & 0.337 & 0.204 & \\
\hline \multicolumn{7}{|l|}{ Brazil Mato Grosso do Sul Nandeva } \\
\hline \multicolumn{7}{|l|}{ Spain Murcia } \\
\hline Spain Majorca & 0.163 & 0.069 & 0.252 & 0.342 & 0.171 & \\
\hline Japan Tokyo & 0.161 & 0.292 & 0.123 & 0.258 & 0.166 & \\
\hline \multicolumn{7}{|l|}{ Brazil Parana Kaingang } \\
\hline Spain Majorca Chueta & 0.199 & 0.117 & 0.16 & 0.289 & 0.192 & \\
\hline Greece Athens & 0.148 & 0.111 & 0.223 & 0.407 & 0.111 & \\
\hline \multicolumn{7}{|l|}{ Brazil Parana Guarani M'byá } \\
\hline \multicolumn{7}{|l|}{ Brazil Mato Grosso do Sul Kaiowa } \\
\hline \multicolumn{7}{|l|}{ Morocco Nador Metalsa } \\
\hline Iran Tehran & 0.083 & 0.167 & 0.167 & 0.472 & 0.111 & \\
\hline Italy Rome & 0.096 & 0.212 & 0.115 & 0.404 & 0.173 & \\
\hline \multicolumn{7}{|l|}{ Argentina Formosa Wichi } \\
\hline \multicolumn{7}{|l|}{ Thailand North East } \\
\hline \multicolumn{7}{|l|}{ Brazil Limao Verde and Corrego Reserve Terena } \\
\hline \multicolumn{7}{|l|}{ Nigeria South Efik } \\
\hline \multicolumn{7}{|l|}{ Argentina Formosa Toba } \\
\hline \multicolumn{7}{|l|}{ USA Caucasian pop2 } \\
\hline Kazakhstan & 0.131 & 0.237 & 0.224 & 0.224 & 0.184 & \\
\hline \multicolumn{7}{|l|}{ USA Boston African American } \\
\hline Saudi Arabia Riyadh & 0.056 & 0.083 & 0.222 & 0.5 & 0.139 & \\
\hline \multicolumn{7}{|l|}{ Japan } \\
\hline \multicolumn{7}{|l|}{ Nigeria South Yoruba } \\
\hline \multicolumn{7}{|l|}{ USA African American pop 2} \\
\hline \multicolumn{7}{|l|}{ Nigeria South Igbo } \\
\hline \multicolumn{7}{|l|}{ China Zhangjiajie Tujias } \\
\hline \multicolumn{7}{|l|}{ China Zhejiang Han } \\
\hline Thailand North East pop 2 & & & & & & \\
\hline
\end{tabular}

Table 1: World populations with the respective allele frequency values for MIC genes A4 to A10. Blank cells indicate the absence of studied conducted. 


\begin{tabular}{|c|c|c|c|c|c|c|c|c|c|c|c|}
\hline & $* 001$ & $* 002$ * & $* 002: 01 * 002: 0$ & $: 02 * 004 * 005$ & $* 006$ & $* 007 * 007: 01$ * & $* 007: 02 * 0$ & $* 008 * 008: 01$ & $* 008: 02 * 009$ & $* 009: 01$ & $* 009: 02 * 010$ \\
\hline China Baotou Han & & & 0.12 & 0.048 & & 0.014 & & 0.231 & 0.005 & 0.12 & 0.0050 .188 \\
\hline \multicolumn{12}{|l|}{ China Guangzhou Han } \\
\hline \multicolumn{12}{|l|}{ China Hui } \\
\hline \multicolumn{12}{|l|}{ China Hunan Province Han } \\
\hline \multicolumn{12}{|l|}{ China North Han } \\
\hline \multicolumn{12}{|l|}{ China Uygur } \\
\hline Brazil Mato Grosso do Sul Nandeva & 0 & & 0.375 & 0 & 0 & 0.011 & & 0.034 & 0 & 0.017 & 00.341 \\
\hline Spain Murcia & 0.078 & 0.16 & & 0.1490 .006 & & 0.01 & & 0.25 & & & 0.045 \\
\hline Spain Majorca & 0.051 & 0.14 & & 0.176 & 0.01 & 0.05 & & 0.29 & 0.09 & & 0.018 \\
\hline \multicolumn{12}{|l|}{ Japan Tokyo } \\
\hline Brazil Parana Kaingang & 0.002 & & 0.396 & $0 \quad 0.02$ & 0 & 0 & 0 & 0.02 & 0 & 0.002 & 00.265 \\
\hline Spain Majorca Chueta & 0.005 & 0.16 & & 0.2 & 0 & 0.68 & & 0.17 & 0.08 & & 0.005 \\
\hline \multicolumn{12}{|l|}{ Greece Athens } \\
\hline Brazil Parana Guarani M'byá & 0 & & 0.215 & 0 & 0 & 0 & 0 & 0.015 & 0 & 0.02 & $\begin{array}{lll}0 & 0.33\end{array}$ \\
\hline Brazil Mato Grosso do Sul Kaiowa & 0 & & 0.641 & 00.003 & 0 & 0 & 0 & 0.006 & 0 & 0.089 & 00.141 \\
\hline Morocco Nador Metalsa & 0.03 & & 0.073 & 0.232 & & 0.024 & & 0.268 & & 0.085 & 0.14 \\
\hline \multicolumn{12}{|l|}{ Iran Tehran } \\
\hline \multicolumn{12}{|l|}{ Italy Rome } \\
\hline Argentina Formosa Wichi & 0 & & 0.476 & 0 & & 0.012 & & 0 & & 0.012 & $0 \quad 0.19$ \\
\hline Thailand North East & 0 & 0.18 & & 0.035 & & 0 & & 0.21 & 0.02 & & 0.182 \\
\hline Brazil Limao Verde and Corrego Reserve Terena & 0 & & 0.445 & 0 & & 0 & & 0.008 & & 0.023 & 00.172 \\
\hline Nigeria South Efik & & & 0.172 & 0.219 & & & & 0.328 & 0.031 & & 0.078 \\
\hline Argentina Formosa Toba & 0.005 & & 0.346 & 0.011 & & 0 & & 0.01 & & 0.016 & 0.0160 .277 \\
\hline USA Caucasian pop2 & 0.03 & 0.13 & & 0.06 & 0 & 0.02 & & 0.55 & 0.03 & & 0.05 \\
\hline \multicolumn{12}{|l|}{ Kazakhstan } \\
\hline USA Boston African American & & & 0.3 & 0.233 & & & & 0.183 & 0.075 & & 0.033 \\
\hline \multicolumn{12}{|l|}{ Saudi Arabia Riyadh } \\
\hline Japan & & 0.15 & & 0.092 & & 0.01 & & 0.3 & 0.17 & & 0.108 \\
\hline Nigeria South Yoruba & & & 0.331 & 0.27 & & & & 0.142 & 0.068 & & 0.007 \\
\hline USA African American pop 2 & & & 0.256 & 0.295 & & & & 0.231 & 0.051 & & 0 \\
\hline Nigeria South Igbo & & & 0.424 & 0.13 & & & & 0.25 & 0.011 & & 0 \\
\hline \multicolumn{12}{|l|}{ China Zhangijajie Tujias } \\
\hline \multicolumn{12}{|l|}{ China Zhejiang Han } \\
\hline Thailand North East pop 2 & & & & & & & & & & & \\
\hline
\end{tabular}

Table 2: World populations with the respective allele frequency values for MICA alleles *001 to *010. Blank cells indicate the absence of studied conducted. 
$* 011 * 012 * 012: 01 * 013 * 014 * 015 * 016 * 017 * 018 * 019 * 020$

China Baotou Han

China Guangzhou Han

China Hui

China Hunan Province Han

China North Han

China Uygur

Brazil Mato Grosso do Sul Nandeva

Spain Murcia

Spain Majorca

Japan Tokyo

Brazil Parana Kaingang

$0.008 \quad 0.002$

0

0.01

$\begin{array}{llll}0 & 0.06 & 0.006 & 0\end{array}$

Spain Majorca Chueta

0.0160 .084

$\begin{array}{llllll}0.02 & 0.02 & 0 & 0 & 0 & 0.17\end{array}$

Greece Athens

Brazil Parana Guarani M'byá

Brazil Mato Grosso do Sul Kaiowa

0

0.03

$0.01 \quad 0.03$

Morocco Nador Metalsa

0

0.03

$\begin{array}{lllll}0 & 0.09 & 0.05 & 0.04 & 0.021\end{array}$

Iran Tehran

Italy Rome

Argentina Formosa Wichi

Thailand North East

Brazil Limao Verde and Corrego Reserve Terena

Nigeria South Efik

Argentina Formosa Toba

USA Caucasian pop2

Kazakhstan

USA Boston African American

Saudi Arabia Riyadh

Japan

0.127

0.035

Nigeria South Yoruba

USA African American pop 2

Nigeria South Igbo

China Zhangjiajie Tujias

China Zhejiang Han

Thailand North East pop 2

Table 3: World populations with the respective allele frequency values for MICA alleles *011 to *020. Blank cells indicate the absence of studied conducted. 


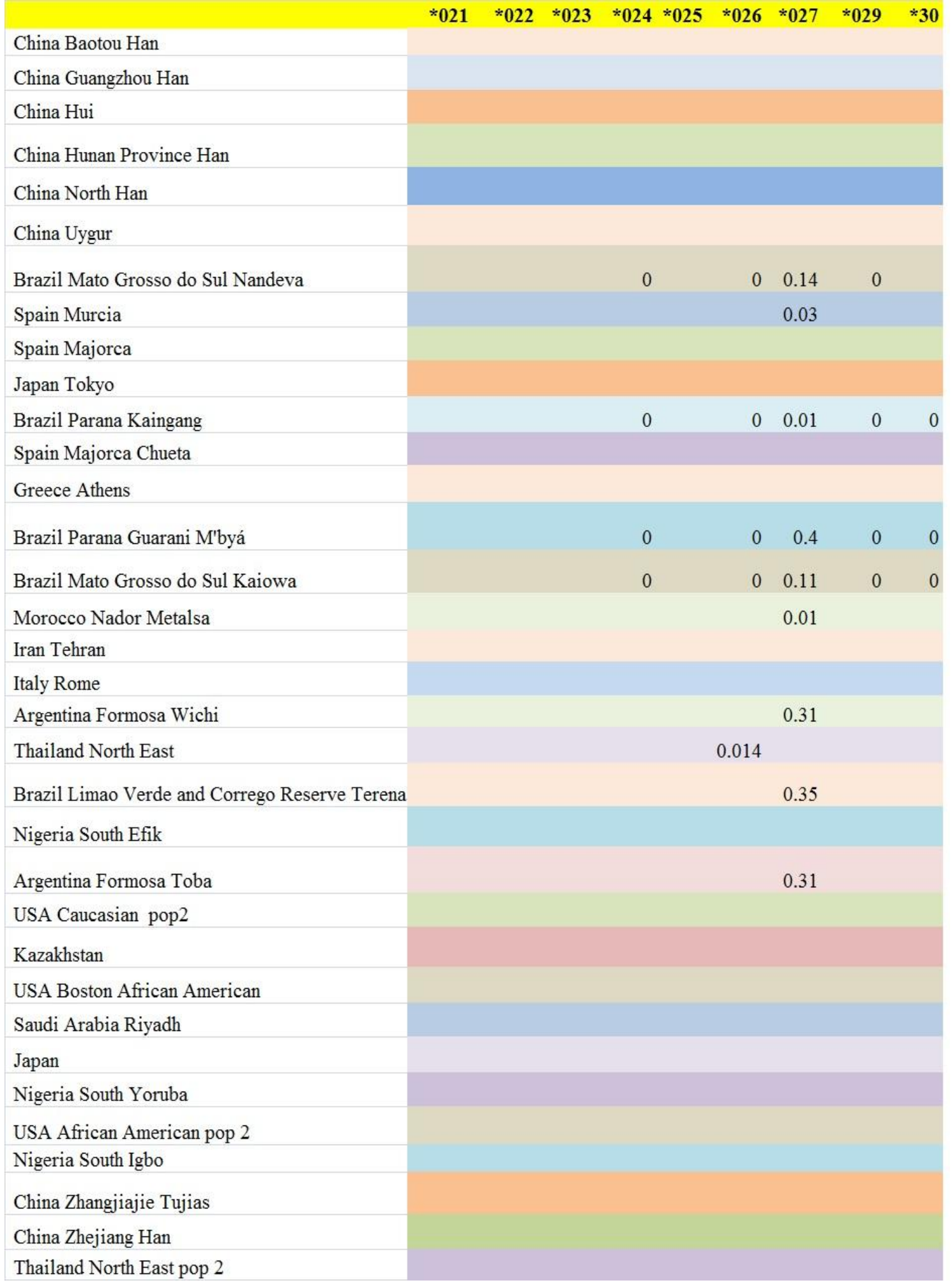

Table 4: World populations with the respective allele frequency values for MICA alleles *021 to *030. Blank cells indicate the absence of studied conducted. 


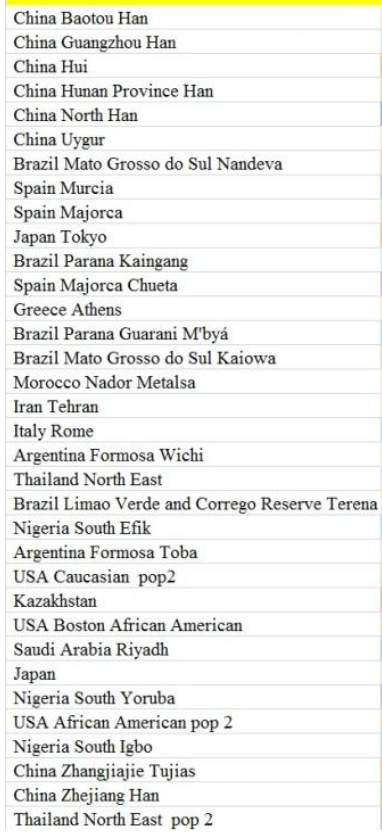

Table 5: World populations with the respective allele frequency values for MICA alleles *030 to *052. Blank cells indicate the absence of studied conducted.

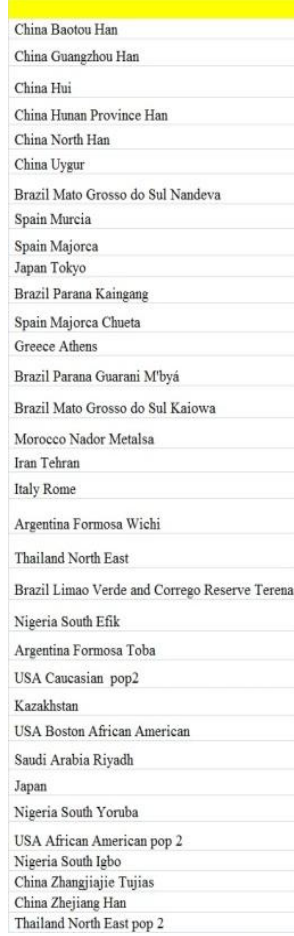

Table 6: World populations with the respective allele frequency values for MICB alleles *001 to *010. Blank cells indicate the absence of studied conducted. 


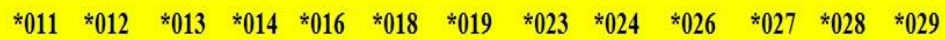

China Baotou Han

China Guangzhou Han

China Hui

China Hunan Province Han

China North Han

China Uygur

Brazil Mato Grosso do Sul Nandeva

Spain Murcia

Spain Majorca

Japan Tokyo

Brazil Parana Kaingang

Spain Majorca Chueta

Greece Athens

Brazil Parana Guarani M'byá

Brazil Mato Grosso do Sul Kaiowa

Morocco Nador Metalsa

Iran Tehran

Italy Rome

Argentina Formosa Wichi

Thailand North East

Brazil Limao Verde and Corrego Reserve Terena

Nigeria South Efik

Argentina Formosa Toba

USA Caucasian pop2

Kazakhstan

USA Boston African American

Saudi Arabia Riyadh

Japan

Nigeria South Yoruba

USA African American pop 2

Nigeria South Igbo

China Zhangjiajie Tujias

China Zhejiang Han

$\begin{array}{lllll}0 & 0.003 & 0 & 0.102 & 0.005\end{array}$

$0.001 \quad 0.039$

$0.02 \quad 0.036$

Thailand North East pop 2

Table 7: World populations with the respective allele frequency values for MICB alleles *011 to *029.Blank cells indicate the absence of studied conducted. 

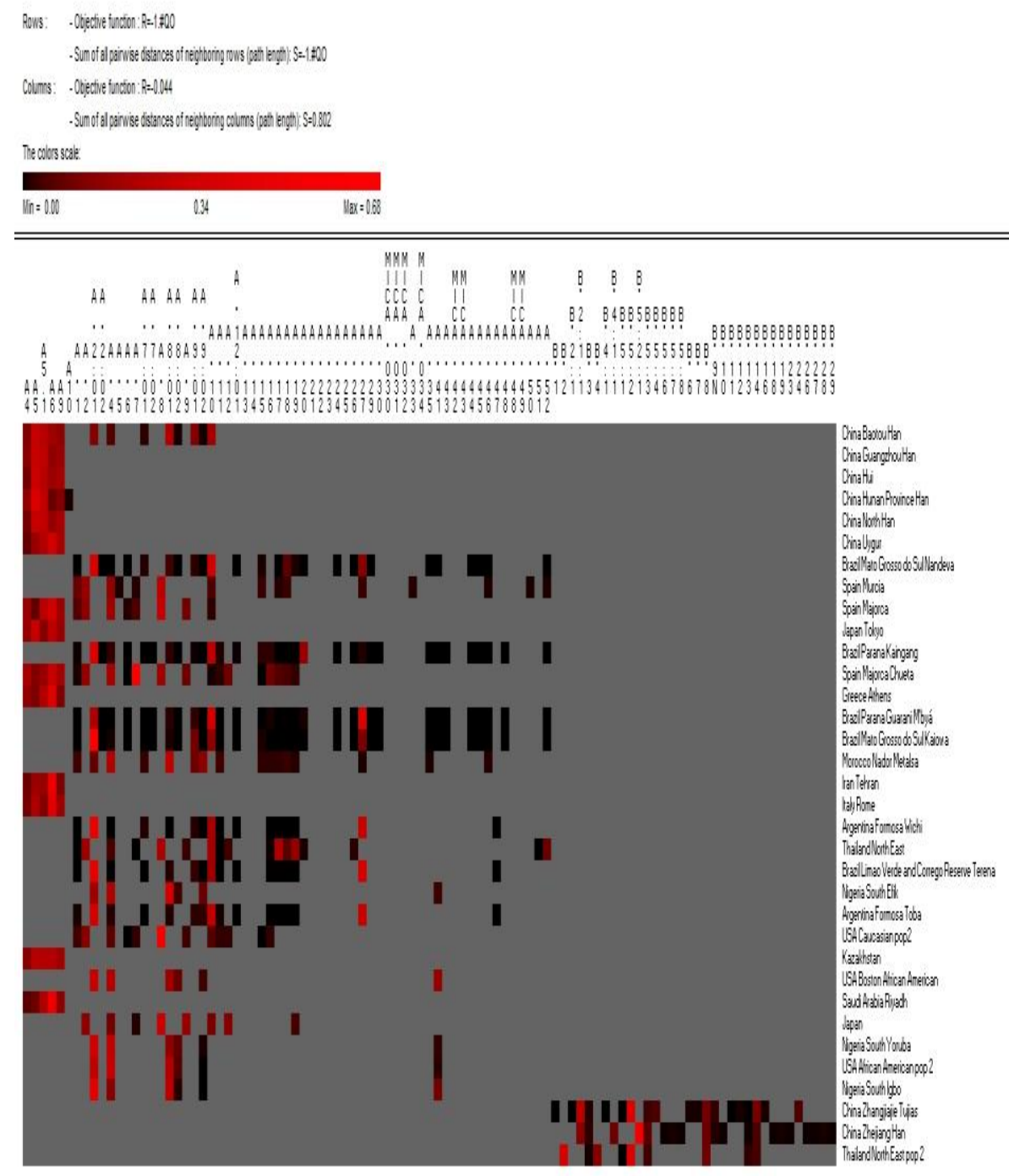

Figure 1: Matrix Plot of the MIC allelic frequencies among the worldwide populations.

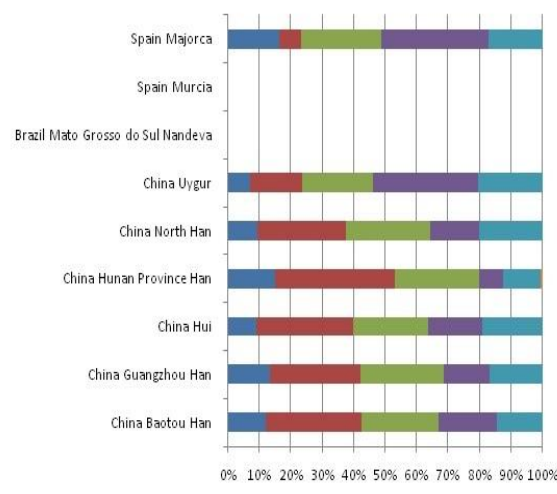

Figure 2

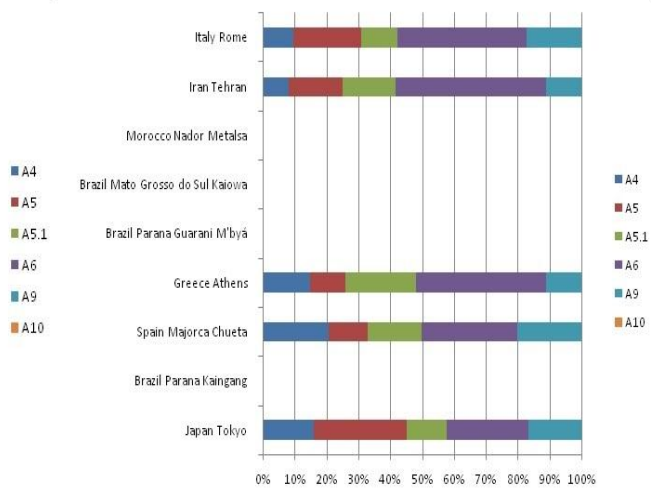

Figure 3 


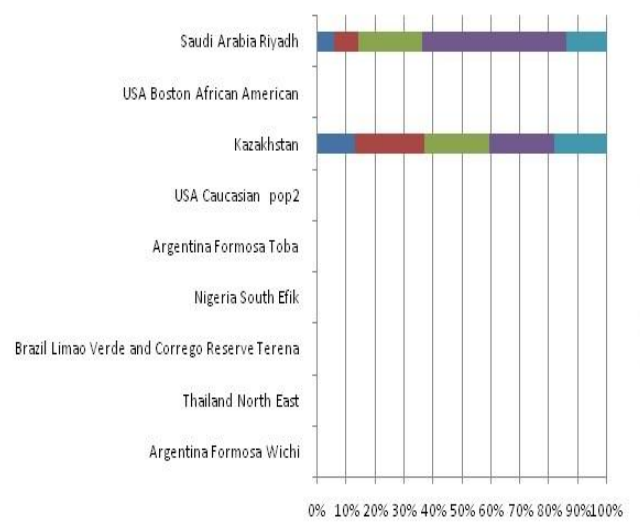

Figure 4

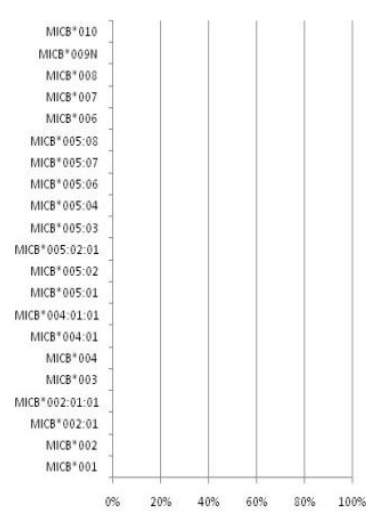

Figure 6

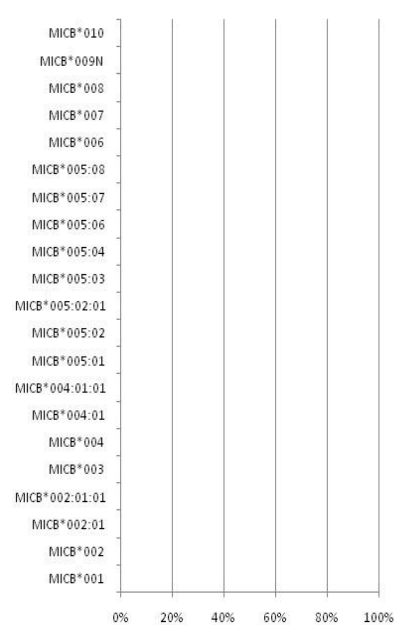

Figure 8

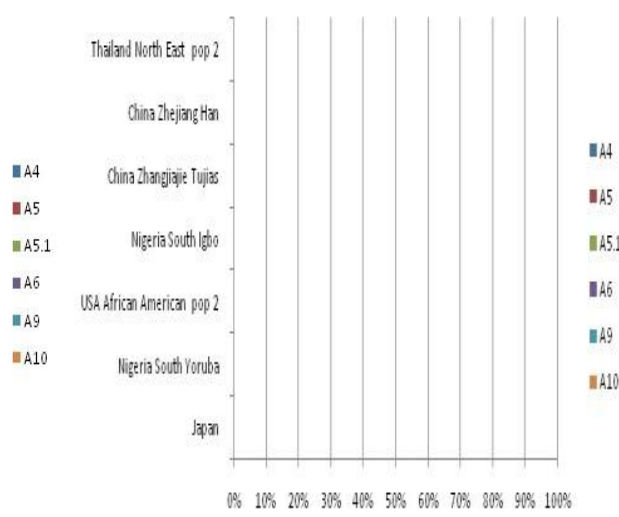

Figure 5

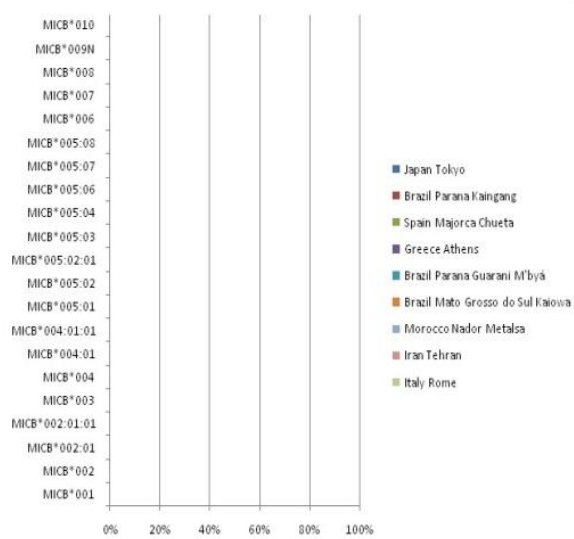

Figure 7

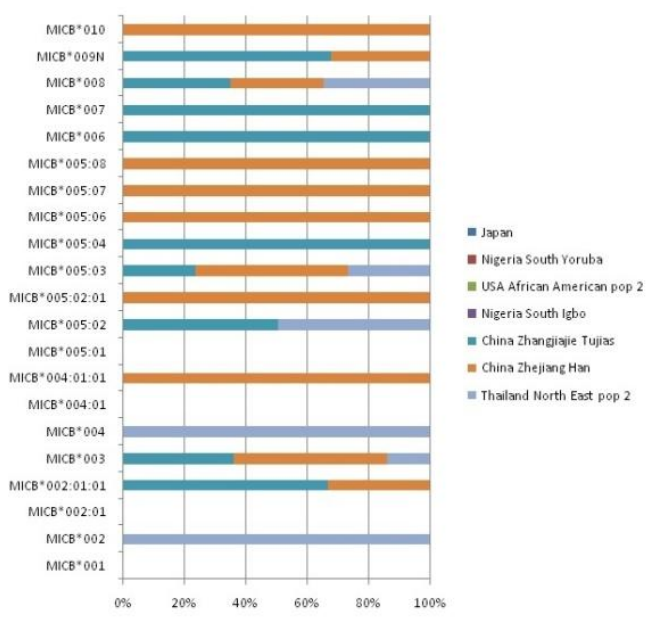

Figure 9 


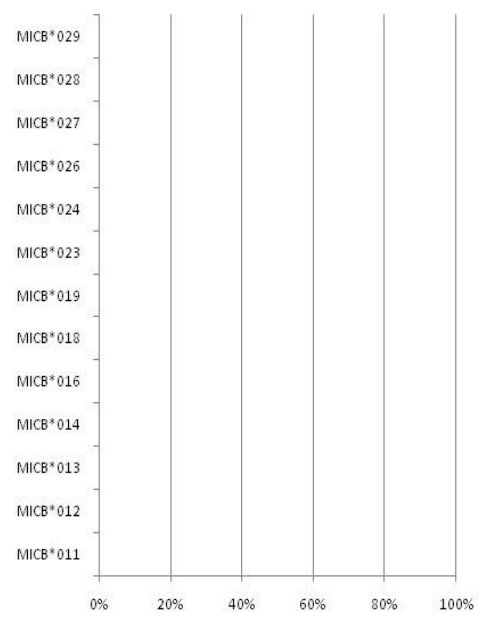

Figure 10

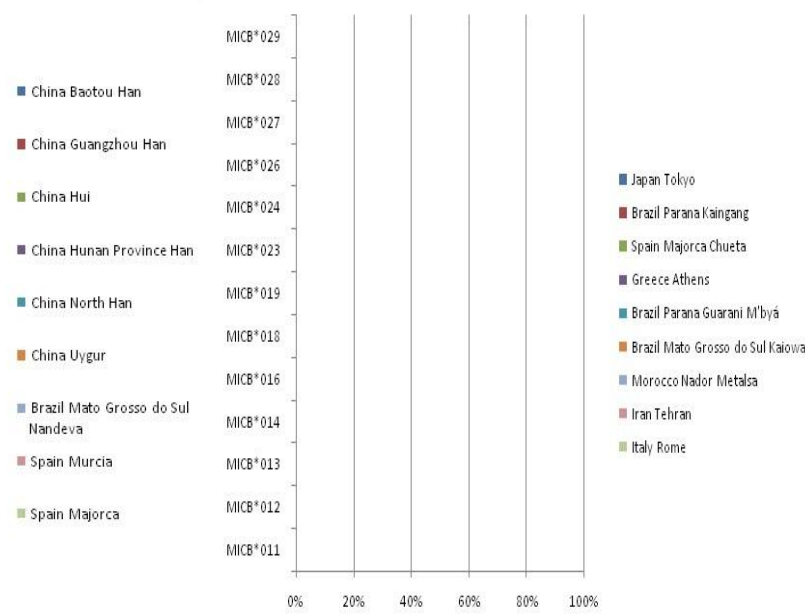

Figure 11

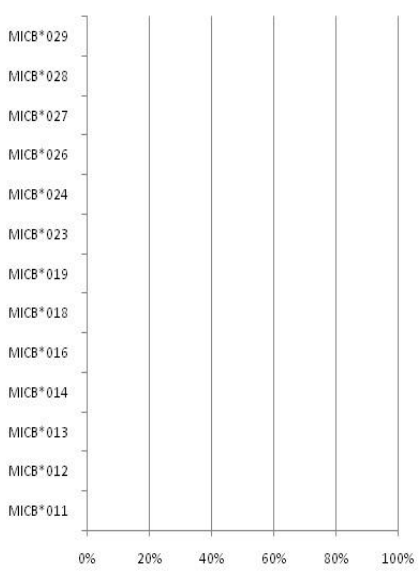

Figure 12

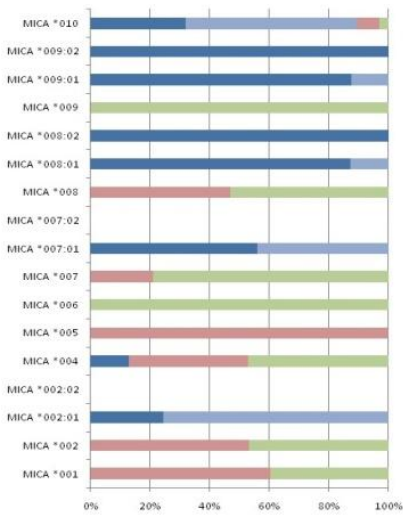

Figure 14
11 Argentina Formosa Wichi

- Thailand North East

Brazil Limao Verde and Corre Reserve Terena

- Nigeria South Efik

- Argentina Formosa Toba

- USA Caucasian pop 2

| Kazakhstan

USA Boston African American

ISaudi Arabia Riyadh

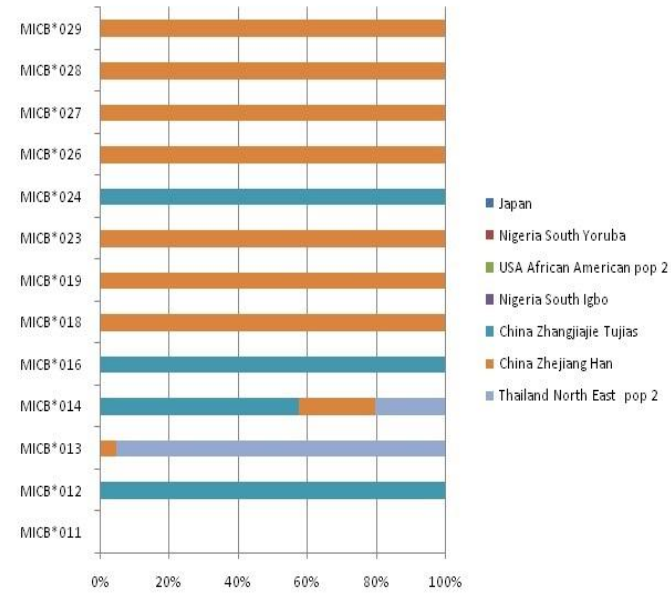

Figure 13

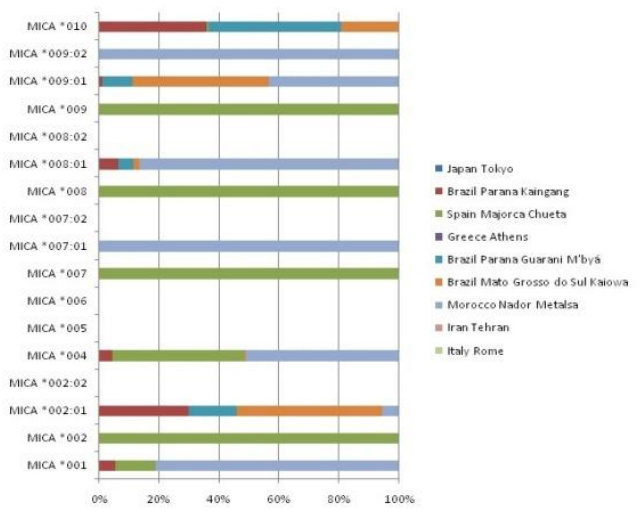

Figure 15 

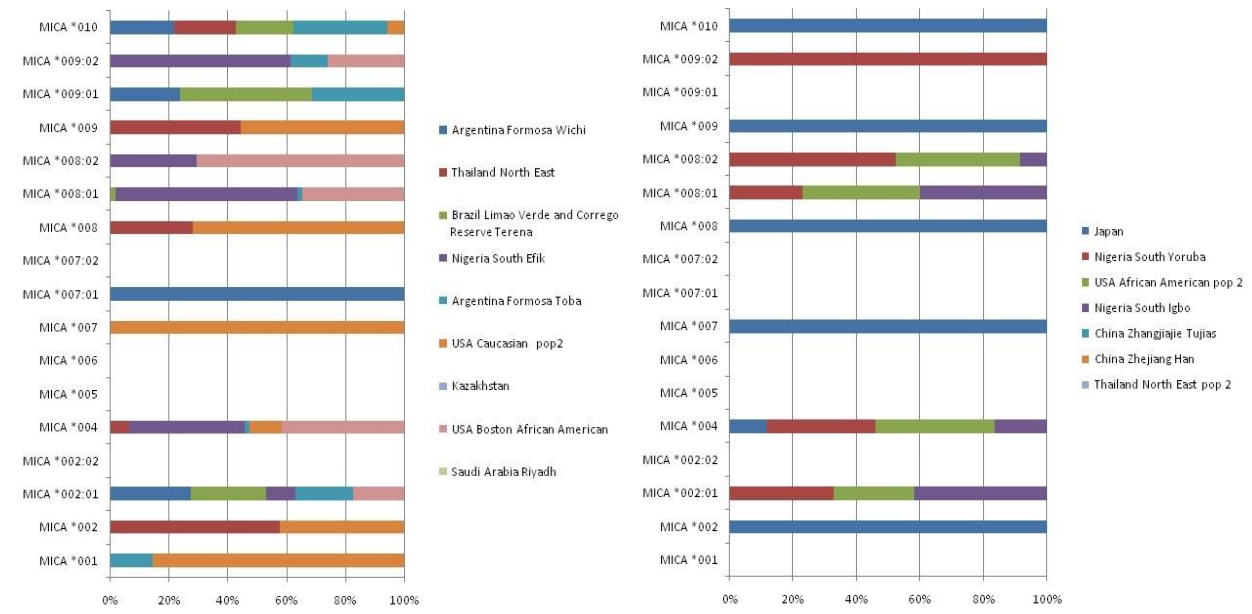

Figure 16

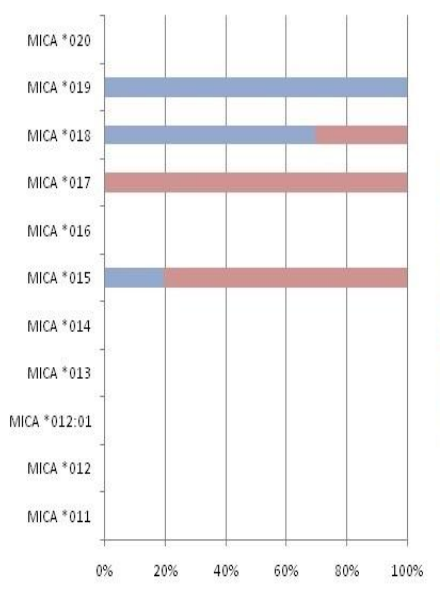

Figure 18

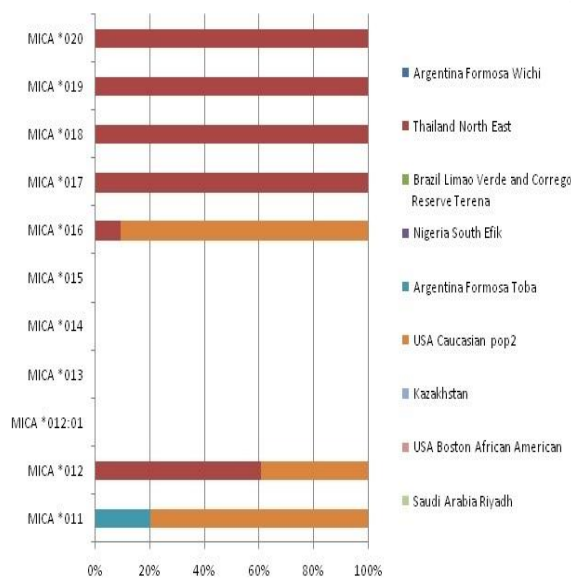

Figure 20

- China Baotou Han

- China Guangzhou Han

II China Hui

- China Hunan Province Han

In China North Han

- China Uygur

II Brazil Mato Grosso do Sul Nandeva

II Spain Murcia

IISpain Majorca$$
\text { MICA }
$$$$
\text { Nic }
$$$$
\text { , }
$$

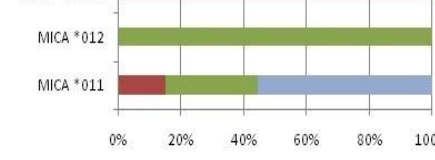

Figure 19

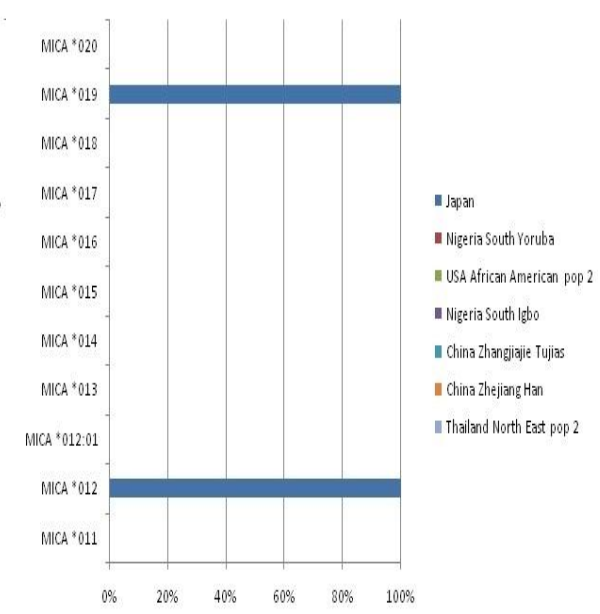

Figure 21 


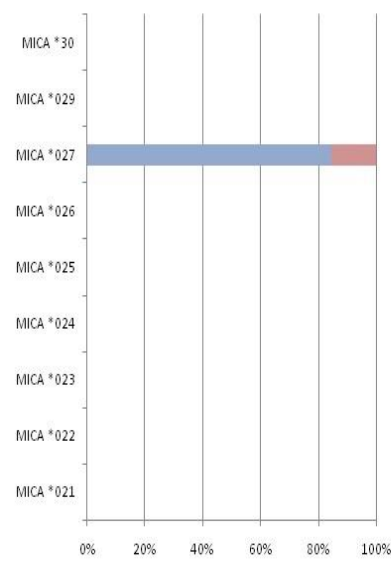

Figure 22

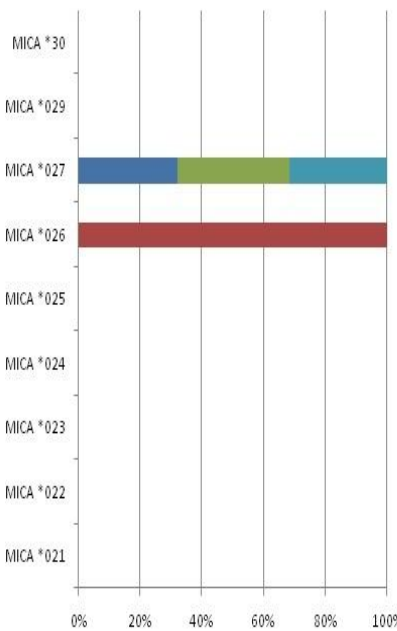

Figure 24

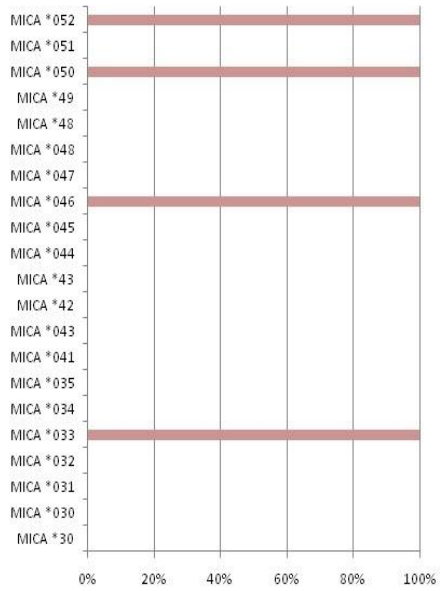

Figure 26

China Baotou Han II China Guanghou Han Ichina Hul - China Hunan Province Han - China North Han II China uygur (11) Brazil Mato Grosso do Sul Nandeva Ispain Murcia IISpain Majorca 22

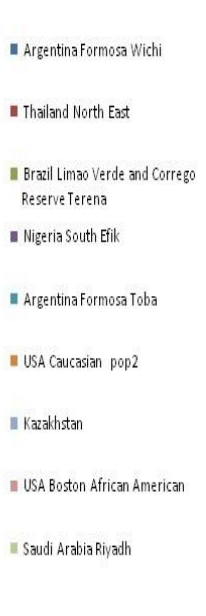

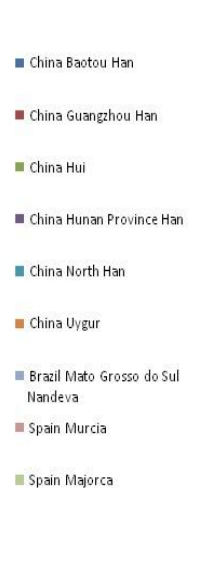

26

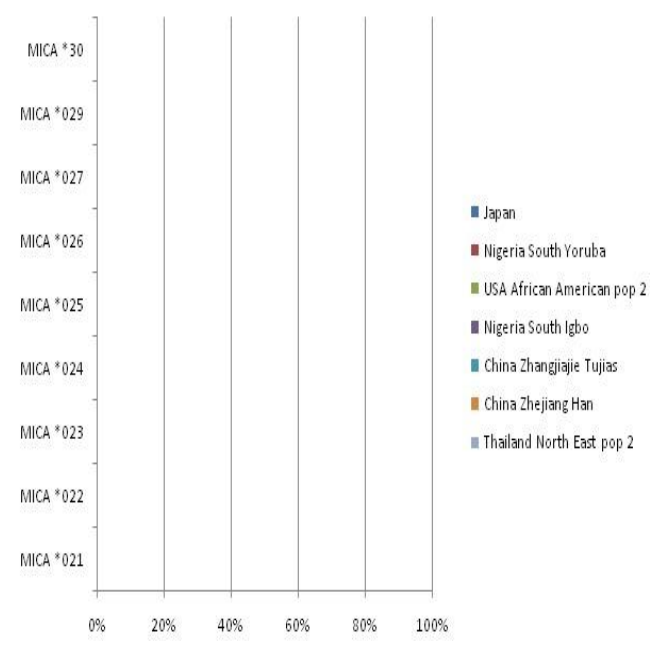

Figure 25

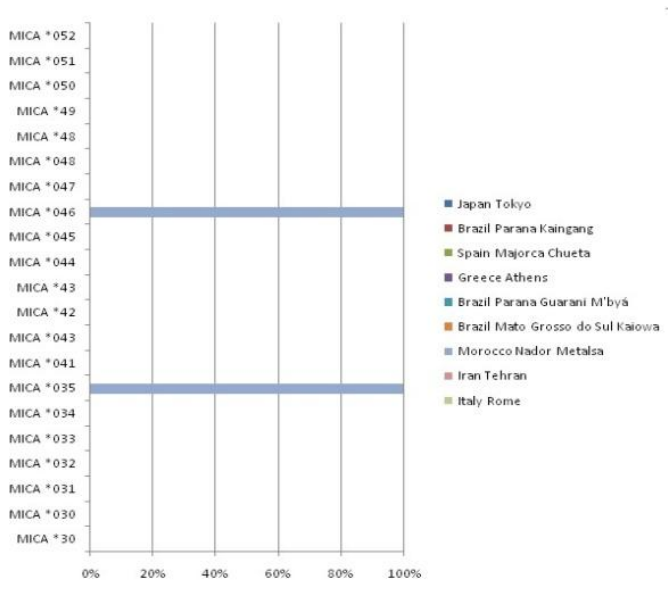

Figure 27 


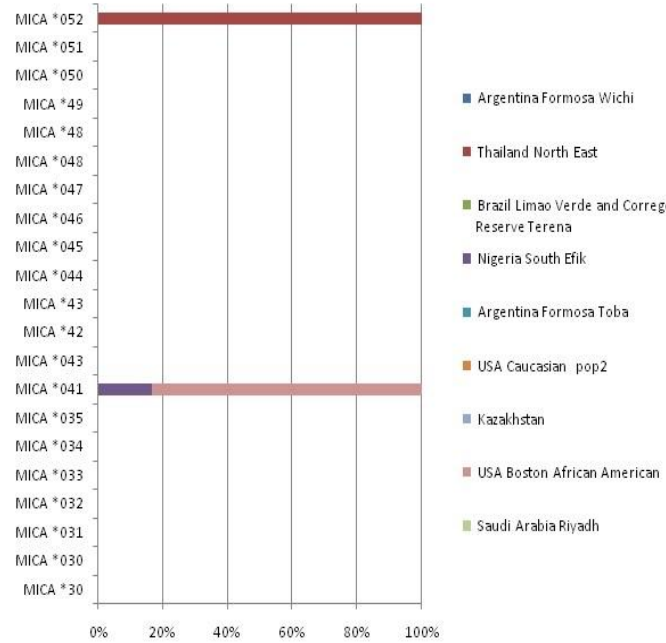

Figure 28 Int J Pharm Biol Sci.

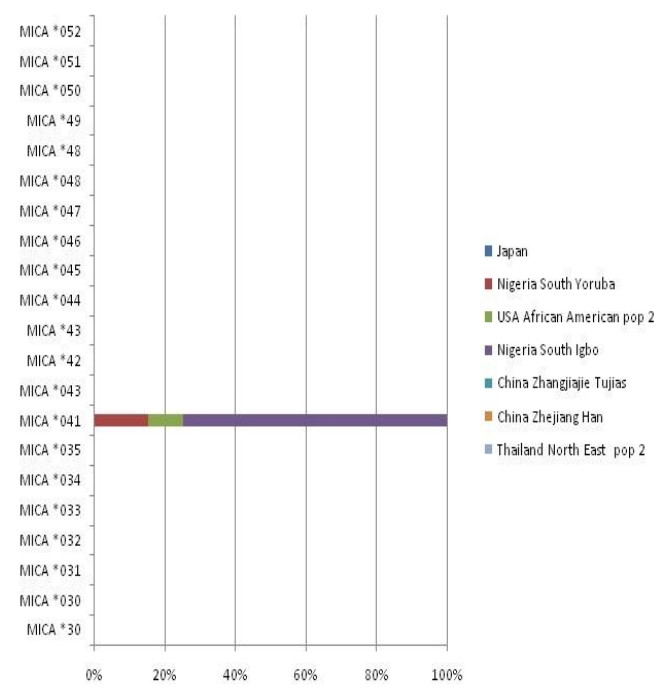

Figure 29

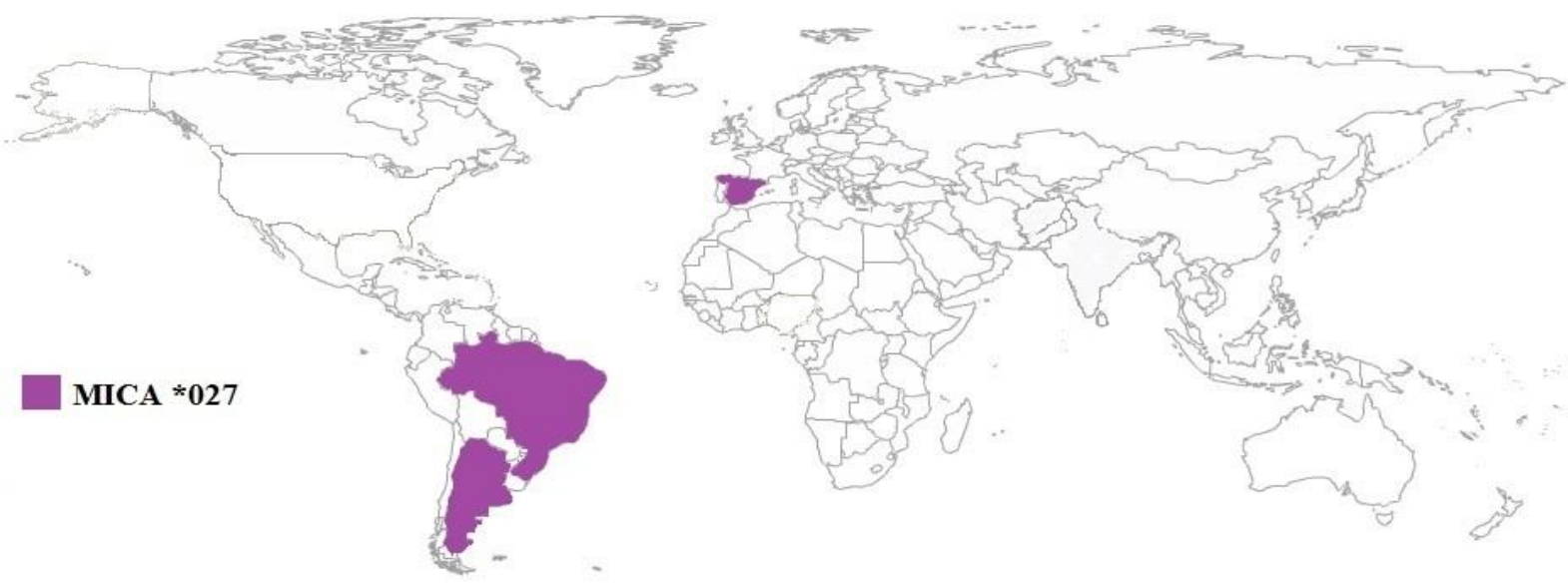

Figure 30: Distribution of the allele MICA *027 among the Spanish and South American populations.

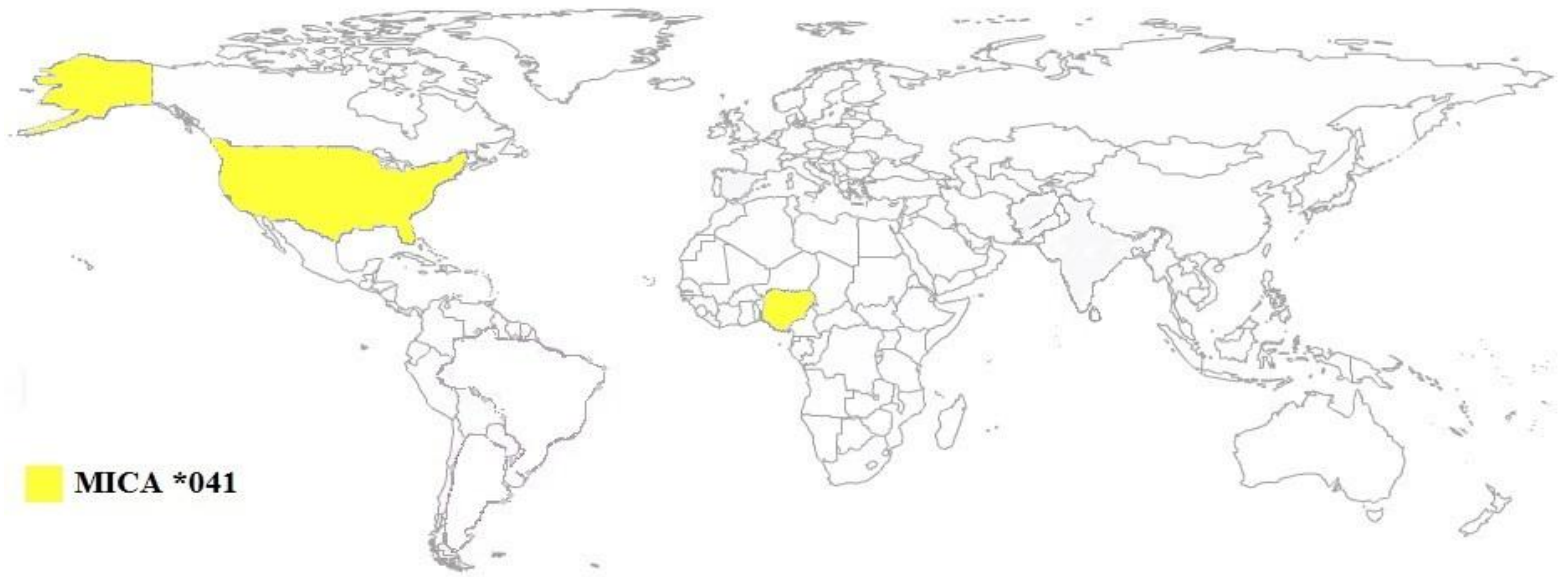

Figure 31: Distribution of the allele MICA *041 among the populations of African origin. 


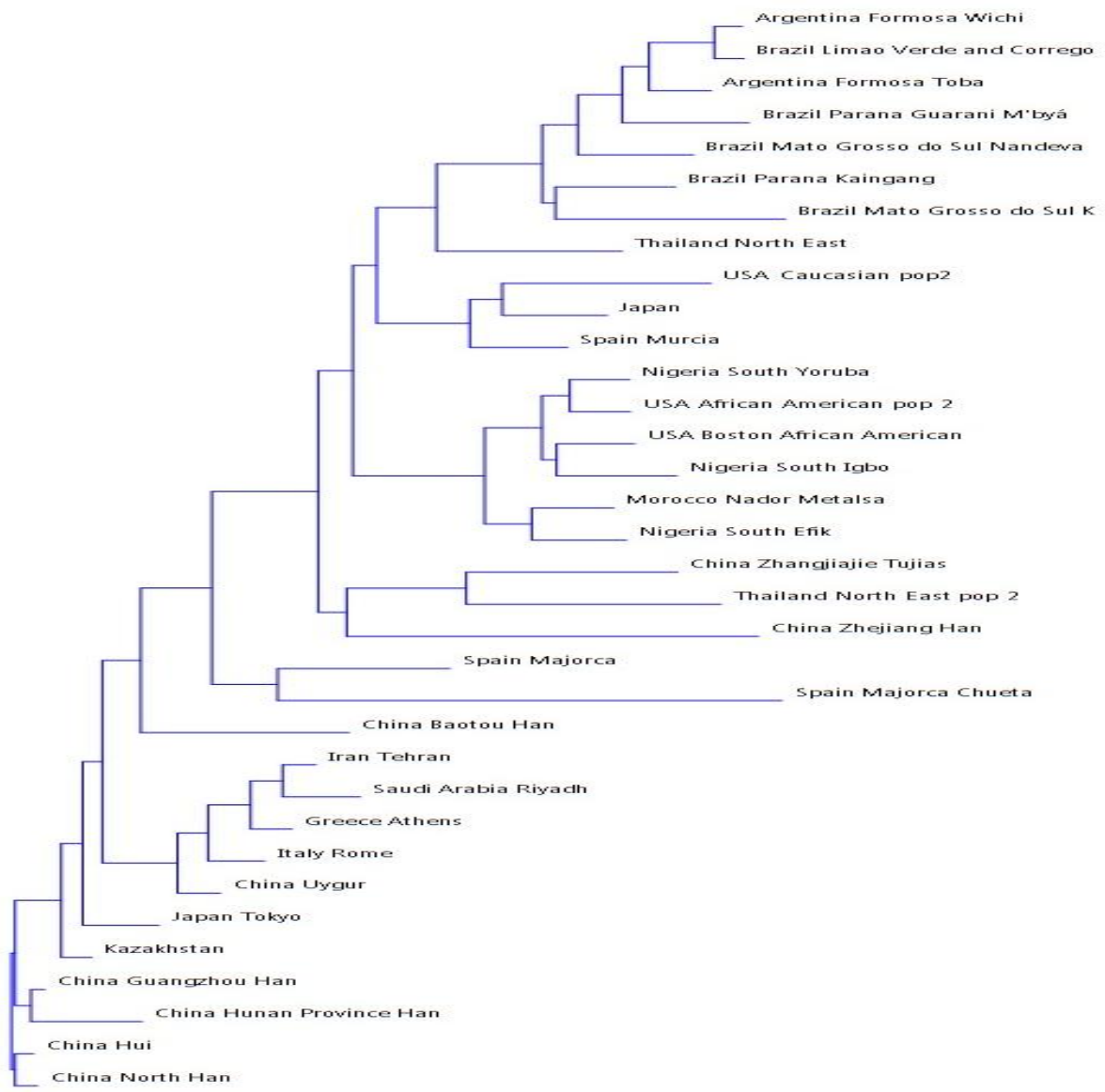

Figure 32a: Neighbor-Joining (NJ)Tree indicating the worldwide populations grouped based on the corresponding MIC A/B allele frequencies.

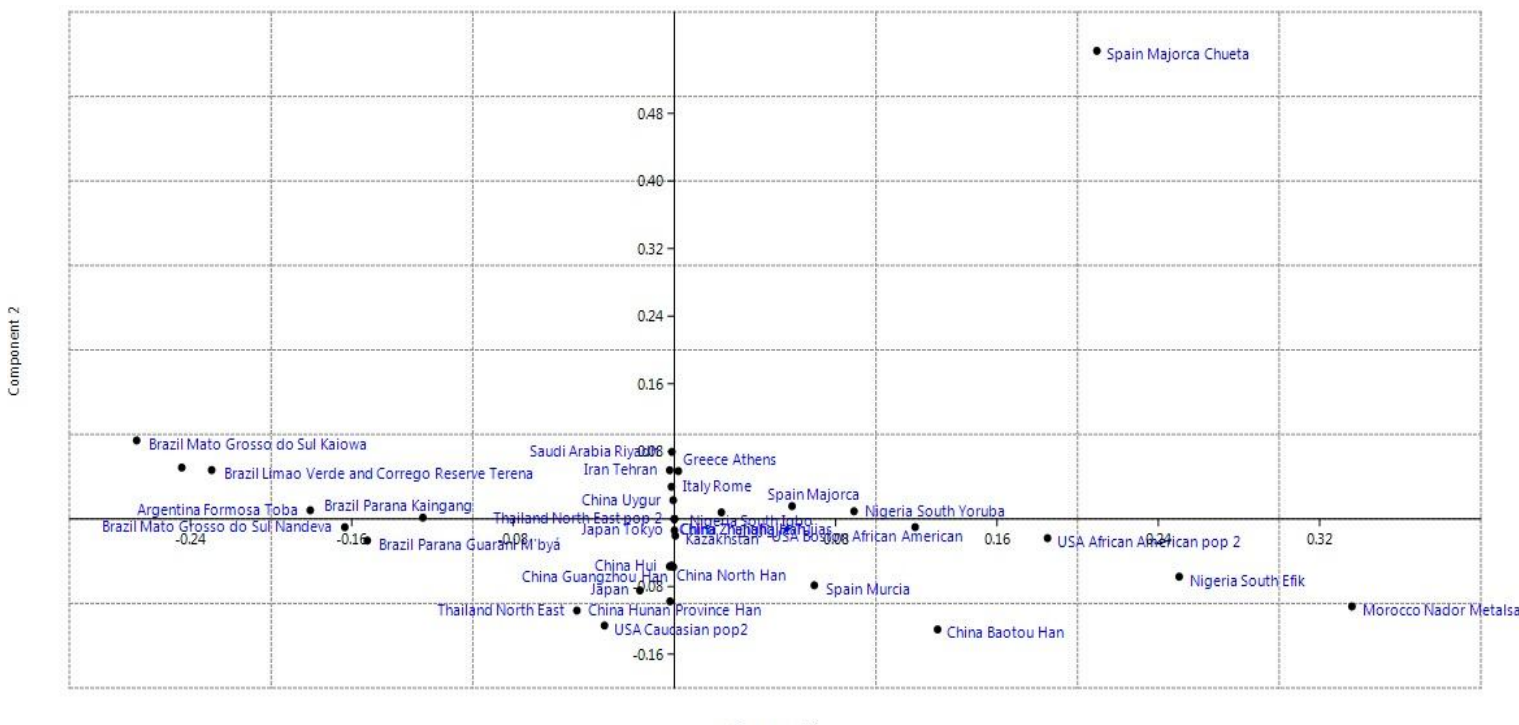

Figure 32b: The Principal component analysis (PCA) graph indicating the worldwide populations clustered based on the corresponding MIC A/B allele frequencies. 


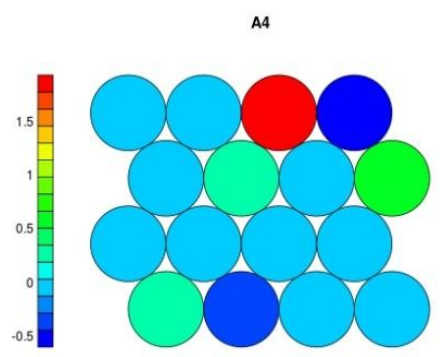

Figure 33

A6

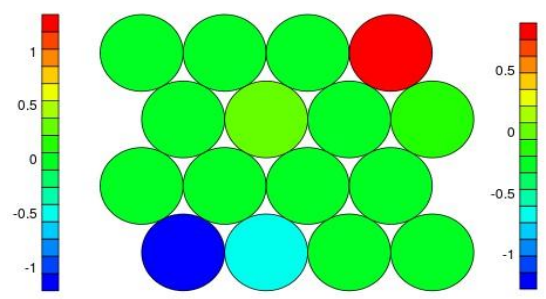

Figure 36

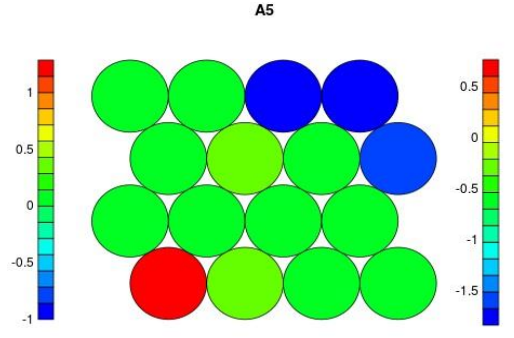

Figure 34

A9

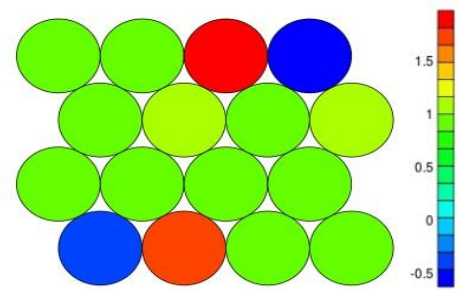

Figure 37

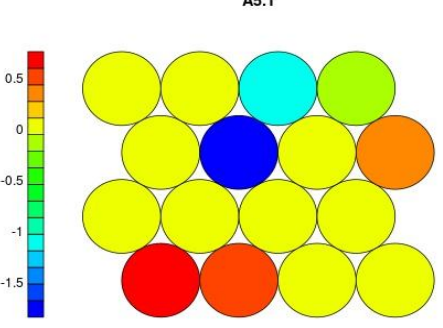

Figure 35

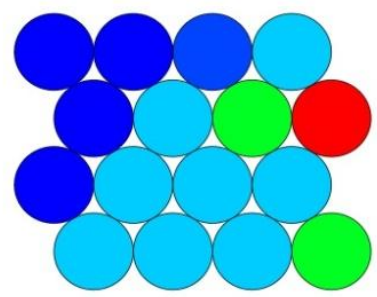

Figure 38

MICA..004

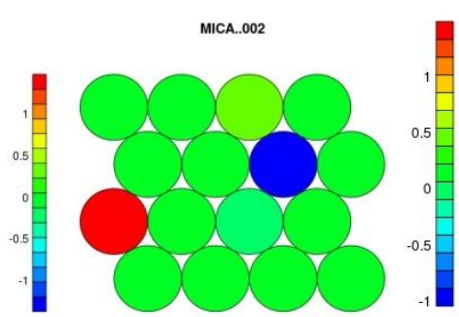

Figure 39

MICA. 007.01

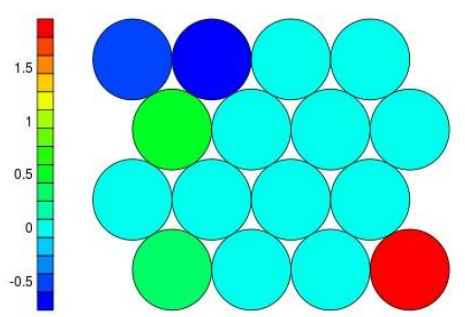

Figure 42

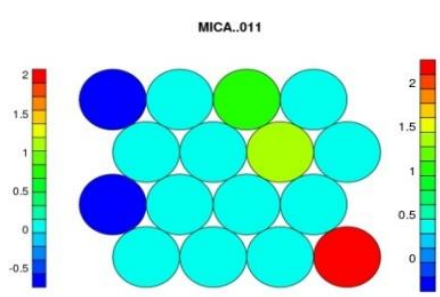

Figure 45

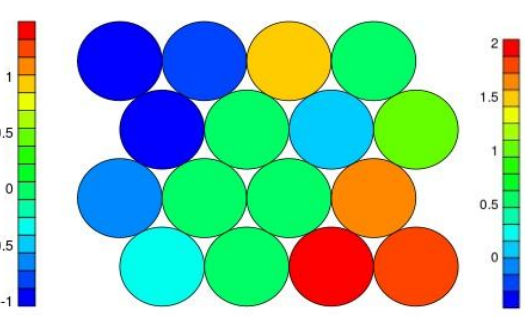

Figure 40

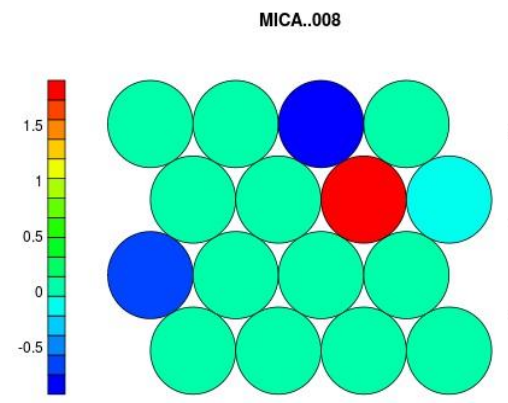

Figure 43

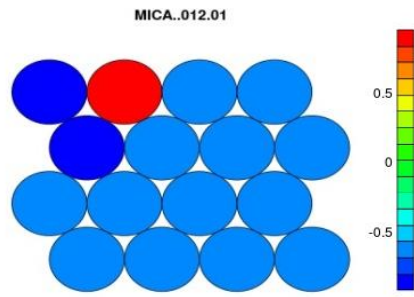

Figure 46

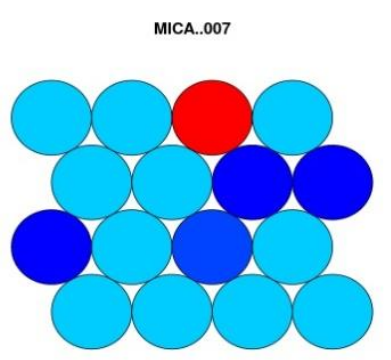

Figure 41

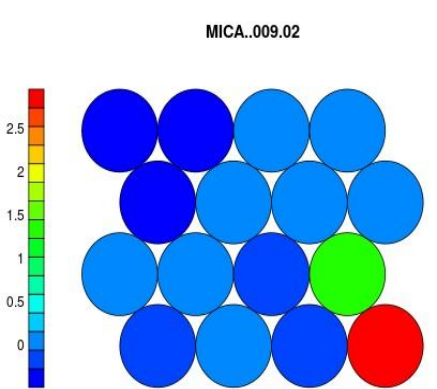

Figure 44

MICA. 015

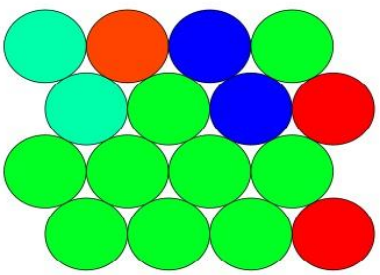

Figure 47 


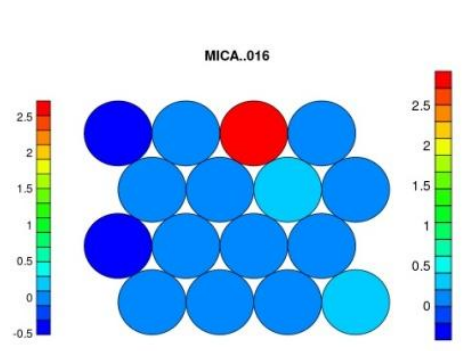

Figure 48

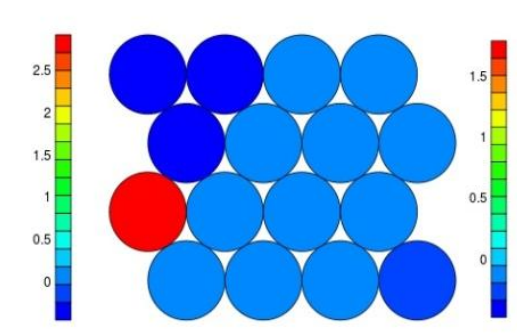

Figure 51

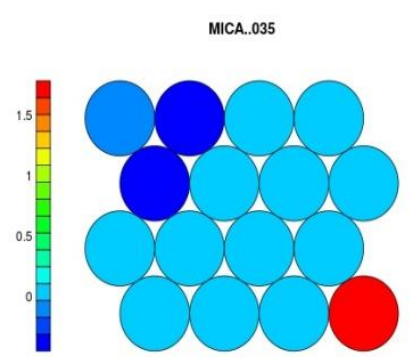

Figure 54

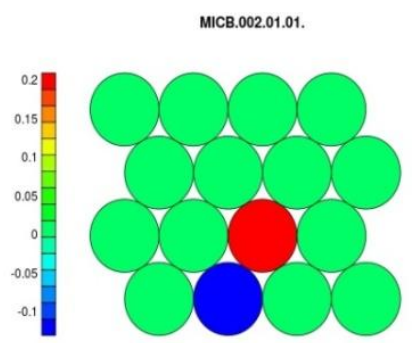

Figure 57
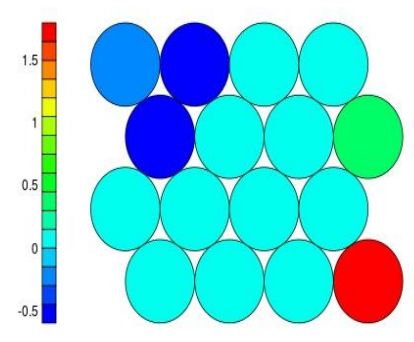

Figure 55

MICB.008.
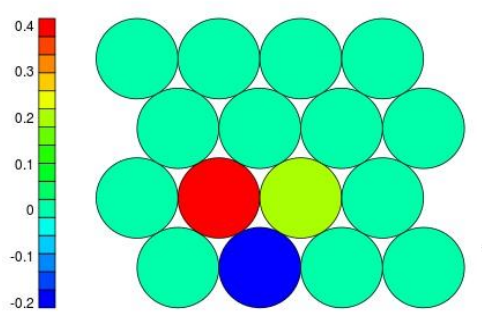

Figure 58

MICB.013.

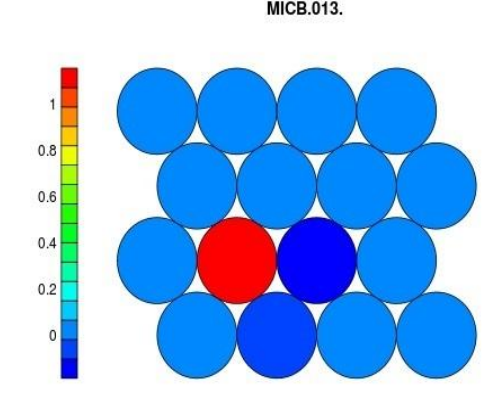

Figure 60
MICA..018

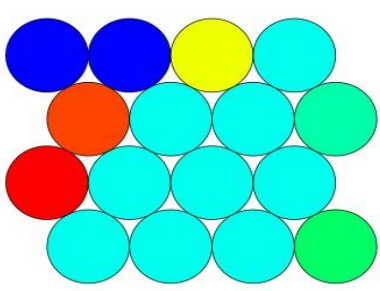

Figure $\mathbf{5 0}$

MICA..026

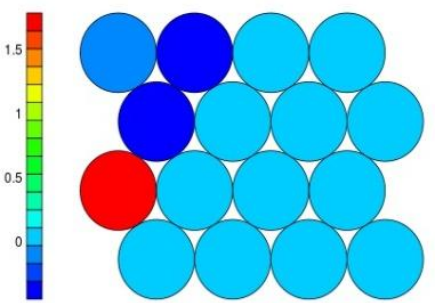

Figure 53

MICA..052

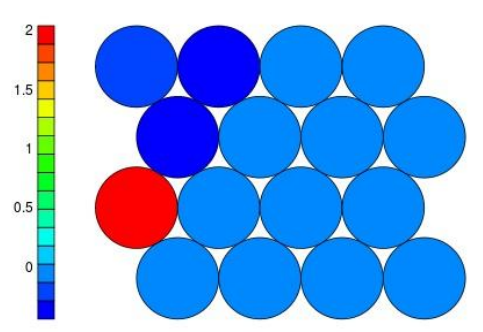

Figure56

MICB.009N.

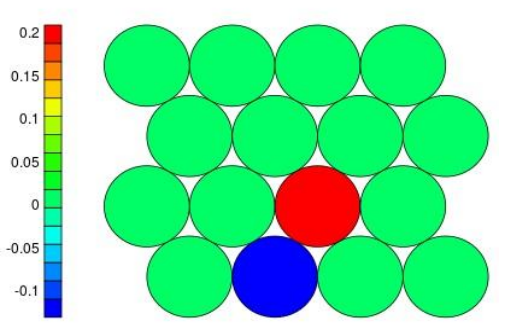

Figure 59

( 


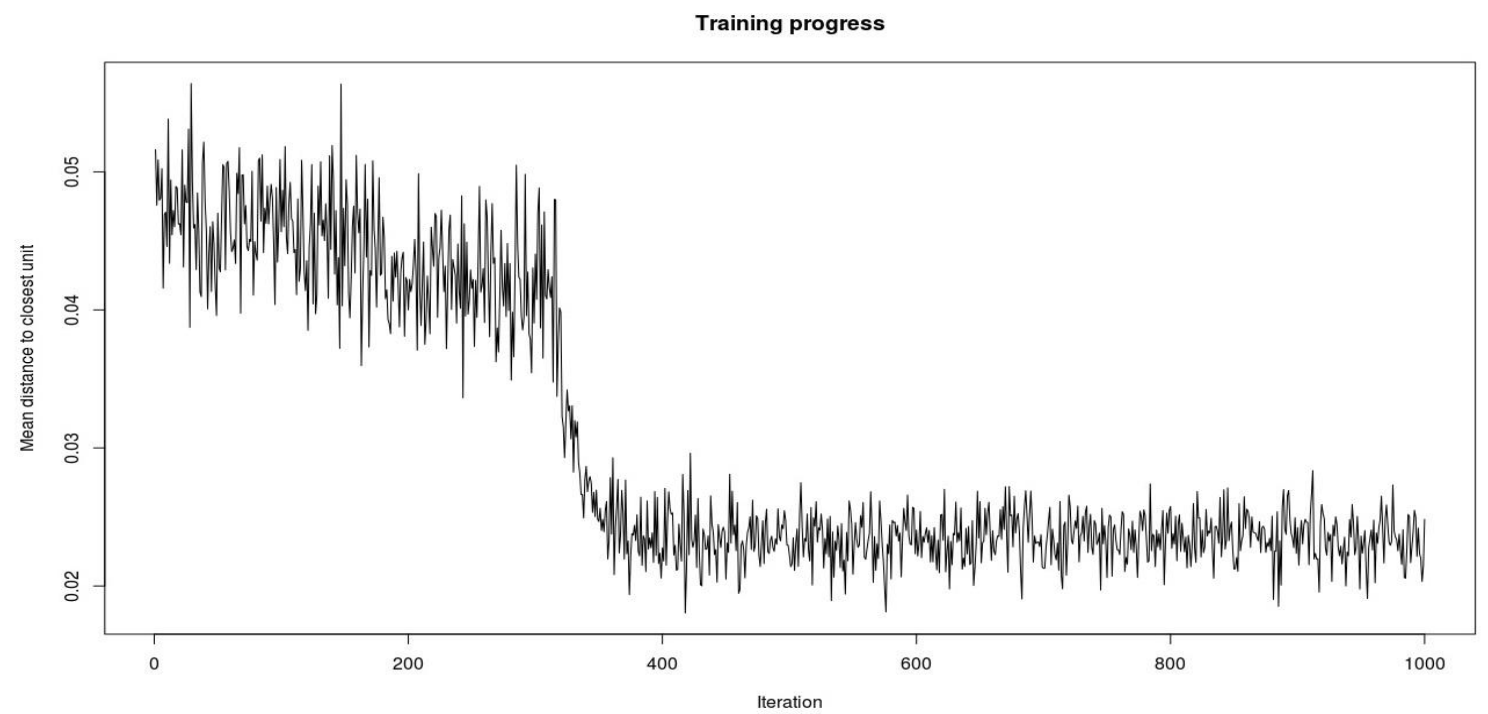

Figure 61: Training graph of the SOM maps.

\section{CONCLUSION:}

Altogether, this study indicates the possibility of including the MIC genes to be the markers for world-wide populations, in addition to the conventional population-genetic markers such as mtDNAs, HLAs and $\mathrm{Y}$ chromosomes. Yet, this study anticipates similar works with different populations, which could give a detailed picture of the distribution of MIC genes worldwide. These results could also complement other studies with genes such as that of HLAs and other markers, to understand the epidemiology of various diseases and importantly of transplantation optimizations.

\section{CONFLICT OF INTERESTS:}

The Authors declare no conflict of Interests.

\section{REFERENCES}

[1]. Ng JHJ, Tachedjian M, Deakin J, Wynne JW, Cui J, Haring $V$, et al, Evolution and comparative analysis of the bat MHC-I region, Nature Publishing Group, (2015),1-18. doi:10.1038/srep21256.

[2] Miller HC, Meally DO, Ezaz T, Amemiya C, Marshall-graves JA, Major Histocompatibility Complex Genes Map to Two Chromosomes in an Evolutionarily Ancient Reptile, the Tuatara Sphenodon punctatus. G3 Journal 5, July (2015):1439-51. doi: 10.1534/g3.115.017467.
[3]. Balasubramaniam S, Bray RD, Mulder RA, Sunnucks P, Pavlova A, Melville J, New data from basal Australian songbird lineages show that complex structure of $\mathrm{MHC}$ class II $\beta$ genes has early evolutionary origins within Passerines, BMC Evolutionary Biology (2016):1-11. doi:10.1186/s12862-016-0681-5.

[4]. Plasil M, Mohandesan E, Fitak RR, Musilova P, Kubickova S, Burger PA, et al, The Major histocompatibility complex in Old world Camelids and low polymorphism of its class II genes, BMC Genomics (2016):1-17. doi:10.1186/s12864-0162500-1.

[5]. Lugo JS, Cadavid LF, Patterns of MHC-G-Like and MHC-B diversification in New World Monkeys, PLoS ONE, (2015).10(6): e0131343. doi:10.1371/journal.pone.0131343.

[6]. Newhouse DJ, Balakrishnan CN, High major histocompatibility complex class I polymorphism despite bottlenecks in wild and domesticated populations of the Zebra finch (Taeniopygia guttata ), BMC Evolutionary Biology (2015):1-11. doi:10.1186/s12862-015-0546-3

[7]. Pechouskova E, Dammhahn M, Brameier $M$, Fichtel C, Kappeler PM, Huchard E, MHC class II variation in a rare and ecological specialist mouse lemur reveals lower allelic richness and contrasting selection patterns compared to a generalist and widespread sympatric congener, Immunogenetics. 67 (2015):229-245. doi: 10.1007/s00251-015-0827-4. 
[8]. Mable BK, Copy number variation and genetic diversity of MHC Class II-b alleles in an alien population of Xenopus laevis, Immunogenetics( 2015):591-603. doi:10.1007/s00251-015-0860-3.

[9]. Grimholt U, MHC and Evolution in Teleosts, Biology, 5(2016) 6. doi:10.3390/biology5010006.

[10]. Liu Y, Li X, Qi J, Zhang N, Xia C, The structural basis of Chicken, Swine and Bovine CD8 $\alpha \alpha$ dimers provides insight into the Co- evolution with MHC I in endotherm species, Nature Publishing Group, April (2016): 1-11. doi:10.1038/srep24788.

[11]. Turner MW, Brostoff J, Wells RS, Stokes CR, HLA in Eczema and Hay fever, Clin. exp. Immunol (1977):43-7.

[12]. Long AE, Gillespie KM, Aitken RJ, Goode JC, Bingley PJ, Williams AJK, Humoral Responses to Islet Antigen-2 and Zinc Transporter 8 Are Attenuated in Patients Carrying HLA-A*24 Alleles at the Onset of Type 1 Diabetes, Diabetes, Vol. 62(2013), June.

[13]. Glenys Thomson, HLA Disease associations: Models for Insulin Dependent Diabetes Mellitus and the Study of Complex Human Genetic Disorders, Annu. Rev. Genet, (1988), 22:31-50.

[14]. Zhou Z, Jensen PE, Structural characteristics of HLA-DQ that may impact DM editing and susceptibility to type-1 diabetes, Frontiers in Immunology, 4(August), (2013):1-6. doi: 10.3389/fimmu.2013.00262.

[15]. Hosomichi K, Mitsunaga S, Nagasaki H, Inoue I, A Bead-based Normalization for Uniform Sequencing depth ( BeNUS ) protocol for Multisamples sequencing exemplified by HLA-B,BMC Genomics,(2014), 15:645.

[16]. Hosomichi K, Jinam TA, Mitsunaga S, Nakaoka H, Inoue I, Phase-defined complete sequencing of the HLA genes by Next-generation sequencing, BMC Genomics, 14(1),(2013):1.

[17]. Yasuda N, Tsuji K, Aizawa M, Itakura K, Inou T, Matsukura $M$, et al, HLA Antigens in Japanese Populations, American Journal of Human Genetics,(1976):390-399.

[18]. Sayad A, Ph D, Akbari MT, Ph D, Pajouhi M, Investigation the role of gender on the HLA-DRB1 and -DQB1 association with Type-1 Diabetes Mellitus in Iranian patients, Cell Journal (Yakhteh), 15(2),(2013):108-15.

[19]. M. J. Greenacre and L. Degos, Correspondence Analysis of HLA Gene Frequency Data from 124
Population Samples, Am J Hum Genet, 29(1977):60-75.

[20]. Ahmed J. Delli, Vaziri-sani F, Lindblad B, Eldinglarsson $\mathrm{H}$, Carlsson A, Forsander $\mathrm{G}$, et al, Zinc Transporter 8 Autoantibodies and their association with SLC3OA8 and HLA-DQ genes differ between immigrant and Swedish patients with newly diagnosed Type 1 Diabetes in the Better Diabetes Diagnosis Study, Diabetes, 61(2012):2556-2564.

[21]. Ward FE, Biegel AA, HL-A Antigens in North American Black Families, American Journal of Human Genetics, 8128(1976):1-8.

[22]. Noble JA, Johnson J, Lane JA, Valdes AM, HLA Class II Genotyping of African American Type 1 Diabetic Patients Reveals Associations Unique to African Haplotypes, Diabetes, ( 2013),62(September).

[23]. Nakaoka H, Mitsunaga S, Hosomichi K, Shyh-yuh $L$, Sawamoto $T$, Fujiwara $T$, et al, Detection of Ancestry Informative HLA alleles confirms the admixed origins of Japanese population, PLos One, (2013),8(4).

[24]. Bahram S, Bresnahan M, Geraghtyt DE, Spies T, A Second lineage of Mammalian major histocompatibility complex class I genes, Proc. Natl. Acad. Sci, 1994;91(July):6259-63.

[25]. Groh V, Bahramtt S, Bauer S, Herman A, Beauchamp $M$, Spies T. Cell stress-regulated human major histocompatibility complex class I gene expressed in Gastrointestinal epithelium, Proc. Natl. Acad. Sci, (1996),93(October):1244550.

[26]. Molinero LL, Fuertes $M B$, Victoria $M$, Fainboim $L$, Rabinovich GA, Costas $A$, et al, NF-kB regulates expression of the MHC Class I-Related Chain A Gene in activated T Lymphocytes , J Immunol,173(2004):5583-5590. doi: 10.4049/jimmunol.173.9.5583.

[27]. Schilling D, Kühnel A, Tetzlaff $F$, Konrad $S$, Multhoff G, NZ28- induced inhibition of HSF1, SP1 and NFK B triggers the loss of the natural killer cell- activating ligands, MICA/B on human tumor cells, Cancer Immunol Immunother, (2015) 64:599-608. doi: 10.1007/s00262-0151665-9.

[28]. Shi P, Yin T, Zhou F, Cui P, Gou S, Wang C, Valproic acid sensitizes Pancreatic cancer cells to natural killer cell-mediated lysis by upregulating MICA and MICB via the PI3K / Akt signaling pathway, BMC Cancer, 2014, 14:370. 
[29]. Okada, Yukinori, Buhm Han, Lam C Tsoi, Philip E Stuart, Eva Ellinghaus, Trilokraj Tejasvi, et al, Fine mapping Major histocompatibility complex associations in Psoriasis and its clinical subtypes, The American Journal of Human Genetics, 95(2014), 162-172, August 7.

[30]. Ayo, Christiane Maria, Ana Vitória, Fábio Batista Frederico, Rubens Camargo Siqueira, Mariana Previato, Fernando Henrique, et al, MHC Class I Chain-Related Gene A Polymorphisms and Linkage Disequilibrium with HLA-B and HLA-C alleles in Ocular Toxoplasmosis, PLOS ONE, 10(12): e0144534. doi:10.1371/journal.pone.0144534.

[31]. Baharav, Ehud, and Abraham Weinberger, The HLA-B * 5101 Molecule-Binding capacity to antigens used in animal models of Behçet's disease: A Bioinformatics Study, IMAJ, (2012), July, Vol 14.

[32]. Wei, Xiaobin, Biqiong Ren, Danqin Lin, Bin Luo, Xianxian $\mathrm{Fu}$, Chunyun $\mathrm{Li}$, and others, Serum soluble Major Histocompatibility Complex Class IRelated Chain A/B expression in Patients with Alcoholic Liver Disease in Hainan Li Community, Int J Clin Exp Med, 8(8),(2015):13928-13936.

[33]. Gong, Zheng, Qi-zhi Luo, Lin Lin, Yu-ping Su, Haibo Peng, Kun Du, and others, Association of MICA Gene Polymorphisms with Liver Fibrosis in Schistosomiasis patients in the Dongting Lake Region, Braz J Med Biol Res, March (2012), Volume 45(3): 222-229. doi: 10.1590/S0100$879 \times 2012007500024$

[34]. Zhou, Xiaodong, Jiucun Wang, Hejian Zou, Michael M Ward, Michael H Weisman, Maribel G Espitia, et al., MICA, A gene contributing strong susceptibility to Ankylosing Spondylitis, Ann Rheum Dis, August(2014),73(8): 1552-1557. doi:10.1136/annrheumdis-2013-203352.

[35]. Allegretti, Yessica L, Constanza Bondar, Luciana Guzman, Eduardo Cueto Rua, Nestor Chopita, Mercedes Fuertes, et al., Broad MICA/B expression in the Small Bowel Mucosa: A link between Cellular stress and Celiac Disease, PLoS ONE, 8(9): e73658. doi:10.1371/journal.pone.0073658.

[36]. Clerc, Sigrid Le, Olivier Delaneau, Cédric Coulonges, Jean-louis Spadoni, Vincent Laville, and Damien Ulveling, Evidence after imputation for a Role of MICA Variants in Nonprogression and Elite Control of HIV Type 1 Infection, JID,(2014):210. doi: 10.1093/infdis/jiu342.
[37]. Chunhua Shi,Hongye Li, Jacob P. Couturier, Karen Yang, Xinjian Guo, Dongyi He, Dorothy E. Lewis and Xiaodong Zhou, Allele specific expression of MICA Variants in human fibroblasts suggests a pathogenic mechanism, The Open Rheumatology Journal, 9(2015): 60-64.

[38]. Mitchell, Anna L, Katie D R Macarthur, Earn H Gan, Lucy E Baggott, Anette S B Wolff, Sophie Bensing, et al., Association of Autoimmune Addison's disease with alleles of STAT4 and GATA3 in European Cohorts', PLoS ONE, 9(3): e88991. doi:10.1371/journal.pone.0088991.

[39]. Wang, Qingwen, and Xiaodong Zhou, Associations of MICA Polymorphisms with Inflammatory Rheumatic Diseases, The Open Rheumatology Journal, 9(2015): 94-100.

[40]. Renbin Yu, Shunyu Xu, Yanxia Wang, Hengjuan Cai, Ping Xu, Role of MICA expression, anti-MICA antibodies and serum MICA during Acute rejection in a Rat-to-Mouse Cardiac transplantation model, Int J Clin Exp Pathol, 8(11),(2015):14514-14520.

[41]. Pierre Tonnerre, Nathalie Gérard, Mathias Chatelais, Caroline Poli, Stéphanie Allard, Sylvie Cury, Céline Bressollette, Anne CesbronGautier, and Béatrice Charreau, MICA Variant Promotes Allosensitization after Kidney Transplantation, J Am Soc Nephrol, May 31; 24(6)(2013): 954-966

[42]. Xie, Jingyan, Mengna Liu, Yujuan Li, Yunzhong $\mathrm{Nie}$, Qiongyu $\mathrm{Mi}$, and Shuli Zhao, Ovarian TumorAssociated microRNA-20a decreases Natural Killer cell cytotoxicity by downregulating MICA / B Expression, Cellular and Molecular Immunology, 11 (2014), 495-502.

[43]. Chen, Yu, Gen Lin, Zeng-qing Guo, Zhi-feng Zhou, Zhi-yong $\mathrm{He}$, and Yun-bin Ye, Effects of MICA expression on the prognosis of advanced Nonsmall cell Lung Cancer and the efficacy of CIK therapy, PLoS ONE, 8(7): e69044. doi:10.1371/journal.pone.0069044.

[44]. Fang, Liang, Jiuyu Gong, Ying Wang, Rongrong Liu, Zengshan Li, Zhe Wang, et al., MICA/B expression is inhibited by Unfolded Protein Response and associated with poor prognosis in human Hepatocellular carcinoma, Journal of Experimental \& Clinical Cancer Research,(2014), 33:76.

[45].Shi P, Yin T, Zhou F, Cui P, Gou S, Wang C, Valproic acid sensitizes pancreatic cancer cells to natural killer cell-mediated lysis by upregulating 
MICA and MICB via the PI3K / Akt signaling pathway, BMC Cancer,(2014), 14:370.

[46]. Ohno, Motoko, Motoyuki Otsuka, Takahiro Kishikawa, and Chikako Shibata, Specific delivery of microRNA93 into HBV-Replicating Hepatocytes downregulates protein expression of Liver cancer susceptible gene MICA, Oncotarget, Vol. 5, No. 14.

[47].Esch, E M G Van, B Tummers, V Baartmans, E M Osse, $N$ Haar, M D Trietsch, and others, Alterations in Classical and Nonclassical HLA expression in recurrent and progressive HPVInduced Usual Vulvar Intraepithelial Neoplasia and Implications for Immunotherapy, Int. J. Cancer, 135 (2014), 830-842.

[48].Siew J, Low Y, Chin YM, Mushiroda T, Kubo M, Govindasamy GK, et al, A Genome Wide Study of Copy Number Variation associated with Nasopharyngeal Carcinoma in Malaysian Chinese Identifies CNVs at $11 \mathrm{q} 14.3$ and $6 \mathrm{p} 21.3$ as Candidate Loci, PLoS ONE, 11(1): e0145774. doi:10.1371/journal.pone.0145774.

[49]. Wei X, Ren B, Lin D, Luo B, Fu X, Li C, et al, Serum soluble major histocompatibility complex class I-related chain A / B expression in patients with alcoholic liver disease in Hainan $\mathrm{Li}$ community, Int J Clin Exp Med, 8(2015):1392813936.

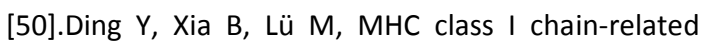
gene $A-A \quad 5^{*} 1$ allele is associated with Ulcerative Colitis in Chinese population, Clinical and Experimental Immunology, 142(2005):193-198. doi:10.1111/j.1365-2249.2005.02907.x.

[51].Tosh K, Ravikumar M, Bell JT, Meisner S, Hill AVS, Pitchappan R, Variation in MICA and MICB genes and enhanced susceptibility to paucibacillary leprosy in South India, Human Molecular Genetics, Vol. 15(2006): 2880-2887. doi:10.1093/hmg/ddl229.

[52].Xavier JM, Davatchi F, Abade O, Shahram F, Francisco V, Abdollahi BS, et al., Characterization of the Major histocompatibility complex locus association with Behçet's disease in Iran, Arthritis Research \& Therapy,17(2015):81. doi:10.1186/s13075-015-0585-6.

[53].Abediankenari S, Yousefzadeh $Y$, Majidi $M$, Ghasemi M, Nasehi MM, MICB Gene expression on Peripheral blood mononuclear cells and susceptibility to Multiple Sclerosis in North of Iran, Iran J Allergy Asthma Immunol, December (2011), 10(4): 261-265.
[54]. Dan Chen, Joanna Hammer, David Lindquist, Annika Idahl \& Ulf Gyllensten, A Variant upstream of HLA-DRB1 and multiple variants in MICA influence susceptibility to Cervical cancer in a Swedish Population, Cancer Medicine, 3(2014): 190-198. doi: 10.1002/cam4.183.

[55]. Woolley N, Mustalahti K, Ma M, Partanen J, Cytokine gene polymorphisms and genetic association with Coeliac Disease in the Finnish Population, Scandinavian Journal of Immunology, 61(2005): 51-56.

[56]. Sung-Hwan Park, Kyung-Su Park, Young-II Seo, DoJune Min, Wan-Uk Kim, Tai-Gyu Kim, Chul-Soo Cho, Jee-Won Mok, Kyung-Sook Park, Ho-Youn Kim, Association of MICA Polymorphism with HLA-B51 and Disease severity in Korean Patients with Behcet's Disease, J Korean Med Sci, 17(2002): 366-70.

[57]. Cho WK, Jung MH, Park SH, Baek IC, Choi H, Kim $\mathrm{T}$, et al, Association of MICA alleles with Autoimmune Thyroid Disease in Korean children, International Journal of Endocrinology, Volume 2012, Article ID 235680, 7 pages. doi:10.1155/2012/235680.

[58]. Campillo JA, López-hernández R, Martínezbanaclocha $H$, Bolarín JM, Gimeno L, Mrowiec A, et al., MHC Class I Chain-Related Gene A Diversity in Patients with Cutaneous Malignant Melanoma from Southeastern Spain, Disease Markers, Volume 2015, Article ID 831864, 6 pages. doi:10.1155/2015/831864.

[59].Puerto F, Nishizawa JE, Kikuchi M, Roca Y, Avilas C, Lora J, et al, Protective Human Leucocyte Antigen Haplotype, HLA- DRB1*01-B*14 against Chronic Chagas Disease in Bolivia, PLoS Negl Trop Dis, 6(3): e1587. doi:10.1371/journal.pntd.0001587.

[60]. Raache R, Belanteur K, Amroun H, Benyahia A, Heniche A, Azzouz M, et al., Association of Major Histocompatibility Complex Class 1 ChainRelated Gene A Dimorphism with Type 1 Diabetes and Latent Autoimmune Diabetes in Adults in the Algerian Population, Clinical and Vaccine Immunology, p. 557-561. doi:10.1128/CVI.05473-11.

[61]. Øyvind Hammer, David A.T. Harper, and Paul D. Ryan, PAST: Paleontological Statistics Software Package for Education and Data analysis, Palaeontologia Electronica,4(1),(2001).9pp. 
[62]. Ron Wehrens and Lutgarde M. C. Buydens, Selfand Super-organizing Maps in R: The kohonen Package, Journal of Statistical software, 2007.

[63]. Joyce Siew Yong Low, Yoon Ming Chin, Taisei Mushiroda, Michiaki Kubo, Gopala Krishnan Govindasamy et al., A Genome Wide Study of Copy Number Variation Associated with Nasopharyngeal Carcinoma in Malaysian Chinese Identifies CNVs at $11 q 14.3$ and $6 p 21.3$ as
Candidate Loci, PLoS ONE, 11(1): e0145774. doi:10.1371/journal.pone.0145774.

[64]. T.R.Orchard, A. Dhar, J. D. Simmons, R. Vaughan, K. I. Welsh and D. P. Jewell, MHC class I chain-like gene $A$ (MICA) and its associations with Inflammatory Bowel Disease and Peripheral Arthropathy, Clin Exp Immunol, 126(2001):437440.
* Corresponding Author: Baburajan Radha Email: babumkubct@gmail.com 